\title{
Novel sum rules for the three-point sector of QCD
}

\author{
A. C. Aguilar ${ }^{1, a}$, M. N. Ferreira ${ }^{1}$, J. Papavassiliou ${ }^{2}$ \\ ${ }^{1}$ University of Campinas, UNICAMP, Institute of Physics “Gleb Wataghin”, Campinas, São Paulo 13083-859, Brazil \\ 2 Department of Theoretical Physics and IFIC, University of Valencia and CSIC, 46100 Valencia, Spain
}

Received: 11 June 2020 / Accepted: 8 September 2020 / Published online: 24 September 2020

(C) The Author(s) 2020

\begin{abstract}
For special kinematic configurations involving a single momentum scale, certain standard relations, originating from the Slavnov-Taylor identities of the theory, may be interpreted as ordinary differential equations for the "kinetic term" of the gluon propagator. The exact solutions of these equations exhibit poles at the origin, which are incompatible with the physical answer, known to diverge only logarithmically; their elimination hinges on the validity of two integral conditions that we denominate "asymmetric" and "symmetric" sum rules, depending on the kinematics employed in their derivation. The corresponding integrands contain components of the three-gluon vertex and the ghost-gluon kernel, whose dynamics are constrained when the sum rules are imposed. For the numerical treatment we single out the asymmetric sum rule, given that its support stems predominantly from low and intermediate energy regimes of the defining integral, which are physically more interesting. Adopting a combined approach based on Schwinger-Dyson equations and lattice simulations, we demonstrate how the sum rule clearly favors the suppression of an effective form factor entering in the definition of its kernel. The results of the present work offer an additional vantage point into the rich and complex structure of the three-point sector of QCD.
\end{abstract}

\section{Introduction}

In recent years, the fundamental $n$-point correlation (Green's) functions of QCD [1] have been the subject of systematic scrutiny both through continuous methods, such as Schwinger-Dyson equations (SDEs) [2-13] and functional renormalization group $[14,15]$, as well as by means of largevolume lattice simulations [16-23]. In this quest, the original intense activity dedicated to the gluon and ghost propagators (two-point sector) [24-51] has been complemented by an indepth exploration of the three-gluon vertex, $\mathbb{\Gamma}_{\alpha \mu \nu}$ [52-64],

a e-mail: aguilar@ifi.unicamp.br (corresponding author) the ghost-gluon vertex, $\Gamma_{\mu}[17,52,57,65-70]$, and, in part, the auxiliary ghost-gluon kernel, $H_{\mu \nu}$ [69]. This concerted effort has catalyzed a vast array of new theoretical insights on the nonperturbative QCD dynamics, and has afforded a tighter grip on a number of complex phenomenological issues [71-80].

As is well-known, the fundamental Slavnov-Taylor identities (STIs) $[81,82]$ impose crucial constraints between the two- and three-point sectors of the theory [83-87]. In the present study, we offer a novel point of view inspired by these profound relations, which, for the special kinematic conditions that we consider, give rise to two relatively simple sum rules.

The starting point of our considerations are certain special projections of $\Gamma_{\alpha \mu \nu}(q, r, p)$, denoted here by $L(q, r, p)$, which have been frequently employed in lattice studies [5961,88-92]. These functions may be evaluated in special kinematic limits, with the final upshot of replacing their three momentum scales by a single one. In particular, in the so-called "asymmetric" and "symmetric" configurations, the resulting quantities, denoted by $L^{\text {asym }}\left(q^{2}\right)$ and $L^{\text {sym }}\left(s^{2}\right)$, respectively, have been computed on the lattice, both in quenched [60-63,92], and unquenched simulations $[59,93]$. The basic quantity evaluated in these cases is $\left\langle\widetilde{A}_{\alpha}^{a}(q) \widetilde{A}_{\mu}^{b}(r) \widetilde{A}_{v}^{c}(p)\right\rangle$, where $\widetilde{A}_{\alpha}^{a}$ are the $\mathrm{SU}(3)$ gauge fields in Fourier space, with the average $\langle\cdot\rangle$ denoting functional integration over the gauge space.

From the continuous standpoint, $L^{\text {asym }}\left(q^{2}\right)$ and $L^{\text {sym }}\left(s^{2}\right)$ may be written as combinations of the form factors appearing in the tensorial decomposition of the pole-free part of $\Gamma_{\alpha \mu \nu}(q, r, p)$, denoted by $\Gamma_{\alpha \mu \nu}(q, r, p)$ [58]. In particular, $L^{\operatorname{asym}}\left(q^{2}\right)$ contains only longitudinal form factors, $X_{i}(q, r, p)$, while $L^{\mathrm{sym}}\left(s^{2}\right)$ involves both longitudinal and transverse form factors, $Y_{i}(q, r, p)$ [94-96], which comprise the terms $L_{L}^{\mathrm{sym}}\left(s^{2}\right)$ and $L_{T}^{\text {sym }}\left(s^{2}\right)$, respectively, with $L^{\mathrm{sym}}\left(s^{2}\right)=L_{L}^{\mathrm{sym}}\left(s^{2}\right)+L_{T}^{\text {sym }}\left(s^{2}\right)$. The nonperturbative extension [58] of the Ball-Chiu (BC) procedure for $\Gamma_{\alpha \mu \nu}(q, r, p)$ [94], in turn, allows one to relate the $X_{i}$ with 
the following quantities: (1) the "kinetic term", $J\left(q^{2}\right)$, of the gluon propagator, (2) the ghost dressing function, $F\left(q^{2}\right)$, and (3) three of the five form factors, $A_{i}(q, p, r)$, comprising $H_{\mu \nu}[69,94,95]$. Of course, this STI-based approach leaves the $Y_{i}$ completely undetermined, since they form the "automatically conserved" part of the three-gluon vertex. Past this point, one introduces theoretical information for the ingredients (1), (2), and (3), entering in these equations, thus obtaining definite predictions for $L^{\text {asym }}\left(q^{2}\right)$ and $L^{\mathrm{sym}}\left(s^{2}\right)$, which are subsequently compared with the corresponding lattice results $[59,63]$.

However, one may reverse this point of view entirely, and consider the relations described above as a means of obtaining $J\left(q^{2}\right)$, once the lattice results for $L^{\text {asym }}\left(q^{2}\right)$ or $L^{\text {sym }}\left(s^{2}\right)$ have been used as inputs. If this alternative perspective is adopted, it becomes immediately clear that these relations may be interpreted as a first-order linear differential equations for $J\left(q^{2}\right)$, whose solution may be written in exact closed form.

It turns out that the general solution of the differential equation associated with $L^{\text {asym }}\left(q^{2}\right)$ displays a simple pole at the origin, while that of $L^{\mathrm{sym}}\left(q^{2}\right)$ exhibits a double one. However, it is well known that the physical $J\left(q^{2}\right)$ does not possess any type of pole at $q^{2}=0$; instead, as it has been established in a series of works, the massless ghost loop entering into the SDE of the $J\left(q^{2}\right)$ forces it to diverge logarithmicaly as $q^{2} \rightarrow 0[58,59,97]$.

These unphysical poles may be eliminated from the solution for $J\left(q^{2}\right)$ by means of an appropriate expansion around the origin, provided that certain integral conditions hold exactly. These conditions will be referred to as the "asymmetric" and "symmetric" sum rule, respectively. At this point, we postpone the determination of $J\left(q^{2}\right)$ from the corresponding solutions, and focus instead on the content and potential applications of these sum rules.

In general, when different sets of ingredients are used as inputs, the sum rules will be satisfied at a varying degree of accuracy, thus providing a quantitative indication of the veracity of the approximations employed for obtaining these ingredients. In that sense, the sum rules may be used as a means of discriminating approximations or truncations schemes, offering hints for their systematic improvement. Such a possibility, in turn, may be especially useful in the field of SDEs, where the absence of a concrete expansion parameter complicates the task of assigning errors to the results obtained or the simplifications implemented.

Expanding on the previous point, it should be clear that, since the sum rules are deduced from the differential equations for $J\left(q^{2}\right)$, the quantities to be probed must be determined from any approach other than the BC solutions themselves. For example, restricting ourselves to the asymmetric case, one may opt for a purely SDE-based analysis, computing both $A_{i}$ and $X_{i}$ from the SDEs of $H_{\mu \nu}$ [69] and
$\Gamma_{\alpha \mu \nu}(q, r, p)[52,54,55,67,98-100]$ respectively, and then use $X_{1}\left(q^{2}\right)$ and $X_{3}\left(q^{2}\right)$ to obtain $L_{S D E}^{\mathrm{asym}}\left(q^{2}\right)$. Alternatively, one may opt for a combined approach, deriving the $A_{i}$ as before, but using lattice data for $L^{\text {asym }}\left(q^{2}\right)[63,64]$; this latter procedure will be followed in the analysis carried out in Sect. 5.

It is important to mention that the integrals defining the two sum rules are evaluated within the interval $\left[0, \mu^{2}\right]$, where $\mu$ denotes the subtraction point where the renormalization of the various Green's functions has been carried out. Consequently, the sum rules explore the quantities entering in them over a wide range of momenta (we use $\mu=4.3 \mathrm{GeV}$ throughout).

In addition, note that the symmetric sum rule involves the contributions from the transverse form factors, $Y_{i}$, which gives rise to the term $L_{T}^{\text {sym }}\left(s^{2}\right)$, introduced earlier. This fact reduces its usefulness, at least within the confines of our approach, because the lattice does not furnish $L_{L}^{\mathrm{sym}}\left(s^{2}\right)$ and $L_{T}^{\text {sym }}\left(s^{2}\right)$ separately, but only their sum. Furthermore, the integrand of the symmetric sum rule contains an extra power of the integration variable, with respect to its asymmetric counterpart; as a result, the support of the ingredients comprising the kernel is suppressed in the low energy regime, which is the most interesting from the nonperturbative point of view. Given the above limitations, for the purposes of this introductory presentation, the numerical analysis will be restricted to the case of the asymmetric sum rule only.

The article is organized as follows. In Sect. 2 we present a brief summary of the main ingredients, originating from the two- and three-point sectors of the theory, that are extensively used in this work. Section 3 is dedicated to the detailed derivation of the central relation for $L^{\text {asym }}\left(q^{2}\right)$, placing particular emphasis on the origin of the special function $\mathcal{W}\left(q^{2}\right)$. Section 4 contains the main results of this study. In particular, after identifying the differential equations and specifying their corresponding solutions, we proceed to the detailed derivation of the two sum rules. In Sect. 5 we demonstrate with a concrete example the possibilities that the asymmetric sum rule offers for constraining one of the ingredients that enter in the expressions defining the function $\mathcal{W}\left(q^{2}\right)$. In Sect. 6 we summarize our results and discuss future applications of the ideas and techniques presented here. We conclude with two Appendices: in the first, we implement the transition from the Taylor scheme to the MOM-type scheme used in the lattice simulations of $L^{\mathrm{sym}}\left(q^{2}\right)$; in the second, we present the steps necessary for the one-loop dressed determination of the function $\mathcal{W}\left(q^{2}\right)$.

\section{Brief review of the main theoretical ingredients}

In this section we introduce the necessary notation and summarize certain basic properties of the two- and three- 
point correlation functions entering in this work. We emphasize that we restrict ourselves to a "quenched" version of QCD, namely a $S U$ (3) Yang-Mills theory with no dynamical quarks; note, in particular, the absence of quark propagators and quark-gluon vertices.

Note that the tensorial structures associated with the aforementioned correlation functions will be written in Minkowski space; final results, when needed, will be passed to the Euclidean space, following standard conversion rules. For the purpose of clarity, in certain places throughout the text, the convention employed ("Minkowski" or "Euclidean") will be stated explicitly.

\subsection{Two-point sector: gluon and ghost propagators}

Throughout this article we work in the Landau gauge, where the gluon propagator $\Delta_{\mu \nu}^{a b}(q)=-i \delta^{a b} \Delta_{\mu \nu}(q)$ is given by the completely transverse form

$$
\Delta_{\mu \nu}(q)=\Delta\left(q^{2}\right) \mathrm{P}_{\mu \nu}(q), \quad \mathrm{P}_{\mu \nu}(q)=g_{\mu \nu}-\frac{q_{\mu} q_{\nu}}{q^{2}}
$$

The special property of infrared saturation displayed by $\Delta\left(q^{2}\right)$, discussed extensively in the literature cited in the Introduction, prompts its splitting into two separate components $[101,102]$, according to

$$
\begin{aligned}
& \Delta^{-1}\left(q^{2}\right)=q^{2} J\left(q^{2}\right)+m^{2}\left(q^{2}\right), \quad \text { [Euclidean] } \\
& \Delta^{-1}\left(q^{2}\right)=q^{2} J\left(q^{2}\right)-m^{2}\left(q^{2}\right), \quad \text { [Minkowski] }
\end{aligned}
$$

where $J\left(q^{2}\right)$ corresponds to the so-called "kinetic term", while $m^{2}\left(q^{2}\right)$ represents a momentum-dependent gluon mass scale, with $m^{2}(0)=\Delta^{-1}(0) \quad[7,10,25,102-115]$. In the ultraviolet, the $J\left(q^{2}\right)$ captures the standard perturbative corrections to $\Delta\left(q^{2}\right)$, while in the infrared it is known to diverge logarithmically [58,59,97].

In addition, we introduce the ghost propagator, $D^{a b}\left(q^{2}\right)=$ $i \delta^{a b} D\left(q^{2}\right)$, and the corresponding dressing function, $F\left(q^{2}\right)$, defined by $D\left(q^{2}\right)=F\left(q^{2}\right) / q^{2}$, which is known to saturate at a finite value in the deep infrared [13,25-27,116].

2.2 Three-point sector: three-gluon vertex, ghost-gluon kernel and vertex

Turning to the three-point sector of the theory, we consider:

1. the three-gluon vertex, $\mathbb{\Gamma}_{\alpha \mu \nu}^{a b c}(q, r, p)=g f^{a b c} \Gamma_{\alpha \mu \nu}$ $(q, r, p)$,

2. the ghost-gluon vertex, $\Gamma_{\mu}^{a b c}(q, p, r)=-g f^{a b c} \Gamma_{\mu}$ $(q, p, r)$,

3. the ghost-gluon kernel, $H_{v \mu}^{a b c}(q, p, r)=-g f^{a b c} H_{\nu \mu}$ $(q, p, r)$.
The diagrammatic representations of these three quantities are given in Fig. 1; all momenta are incoming, $q+p+r=0$.

Regarding $\Gamma_{\mu}(q, p, r)$ and $H_{v \mu}(q, p, r)$, note first that they are related by the STI $q^{v} H_{\nu \mu}(q, p, r)=\Gamma_{\mu}(q, p, r)$. Their respective tensorial decompositions are given by [69, $94,95]$

$$
\begin{aligned}
\Gamma_{\mu}(q, p, r)= & q_{\mu} B_{1}(q, p, r)+r_{\mu} B_{2}(q, p, r), \\
H_{\nu \mu}(q, p, r)= & g_{\mu \nu} A_{1}+q_{\mu} q_{\nu} A_{2}+r_{\mu} r_{\nu} A_{3} \\
& +q_{\mu} r_{\nu} A_{4}+r_{\mu} q_{\nu} A_{5}
\end{aligned}
$$

where the argument $(q, p, r)$ of the $A_{i}$ has been suppressed for compactness. At tree-level, $B_{1}^{(0)}=1$ and $B_{2}^{(0)}=0$, while $A_{1}^{(0)}=1$ and $A_{i}^{(0)}=0$, for $i=2, \ldots, 5$. In addition, it is convenient to introduce the short-hand notation

$A_{d}(q, p, r):=A_{3}(q, p, r)-A_{4}(q, p, r)$.

We next consider $\Gamma_{\alpha \mu \nu}(q, r, p)$, which is of central importance for what follows. Note that, within the framework composed by the pinch-technique (PT) [7, 104, 109] and the background-field method (BFM) [117] [denominated "PTBFM scheme"], $\Gamma_{\alpha \mu \nu}(q, r, p)$ must contain a special component, $V_{\alpha \mu \nu}$, comprised by longitudinally coupled massless poles, i.e., of the form

$$
\begin{aligned}
V_{\alpha \mu \nu}(q, r, p)= & \left(\frac{q_{\alpha}}{q^{2}}\right) A_{\mu \nu}(q, r, p)+\left(\frac{r_{\mu}}{r^{2}}\right) B_{\alpha \nu}(q, r, p) \\
& +\left(\frac{p_{v}}{p^{2}}\right) C_{\alpha \mu}(q, r, p)
\end{aligned}
$$

As has been explained in [31], these poles emerge dynamically, through the formation of (colored) massless boundstate excitations [103,118-121], governed by a special Bethe-Salpeter equation. When inserted into the SDE of the gluon propagator, the term $V_{\alpha \mu \nu}(q, r, p)$ triggers the wellknown Schwinger mechanism [122,123], accounting eventually for the fact that $\Delta^{-1}(0)=c>0$. Note that, due to the property $P_{\alpha \alpha^{\prime}}(q) P_{\mu \mu^{\prime}}(r) P_{\nu v^{\prime}}(p) V^{\alpha \mu \nu}(q, r, p)=0$, the $V_{\alpha \mu \nu}(q, r, p)$ is undetected by transversely projected Green's functions, or lattice "observables" (see Eq. (3.1) below).

The presence of $V_{\alpha \mu \nu}(q, r, p)$ inside $\Gamma_{\alpha \mu \nu}(q, r, p)$, in conjunction with Eq. (2.2), induces a special realization of the fundamental STI [94]

$$
\begin{aligned}
q^{\alpha} \Gamma_{\alpha \mu \nu}(q, r, p)= & F\left(q^{2}\right)\left[\Delta^{-1}\left(p^{2}\right) \mathrm{P}_{\nu}^{\alpha}(p) H_{\alpha \mu}(p, q, r)\right. \\
& \left.-\Delta^{-1}\left(r^{2}\right) \mathrm{P}_{\mu}^{\alpha}(r) H_{\alpha \nu}(r, q, p)\right]
\end{aligned}
$$

and its cyclic permutations. Specifically, writing

$\Gamma_{\alpha \mu \nu}(q, r, p)=\Gamma_{\alpha \mu \nu}(q, r, p)+V_{\alpha \mu \nu}(q, r, p)$,

where $\Gamma^{\alpha \mu v}(q, r, p)$ denotes the remainder of the three-gluon vertex, which does not contain poles, one has $[10,31,102$, 


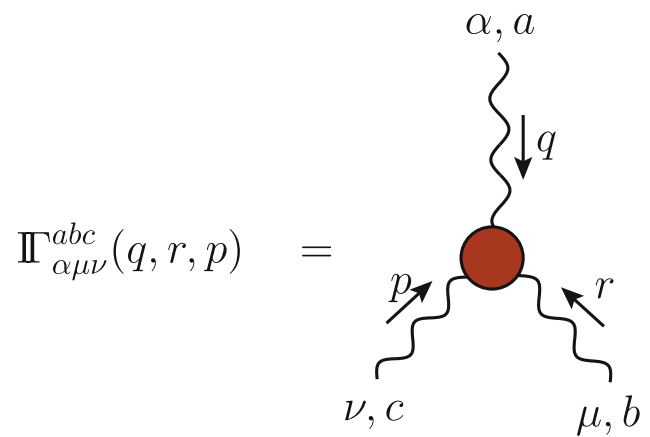

(a)

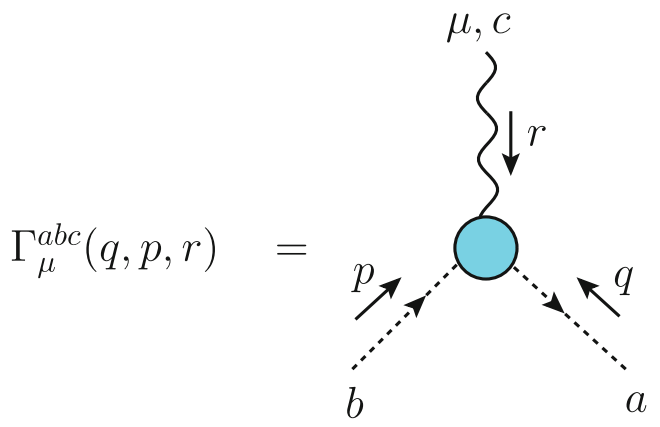

(b)

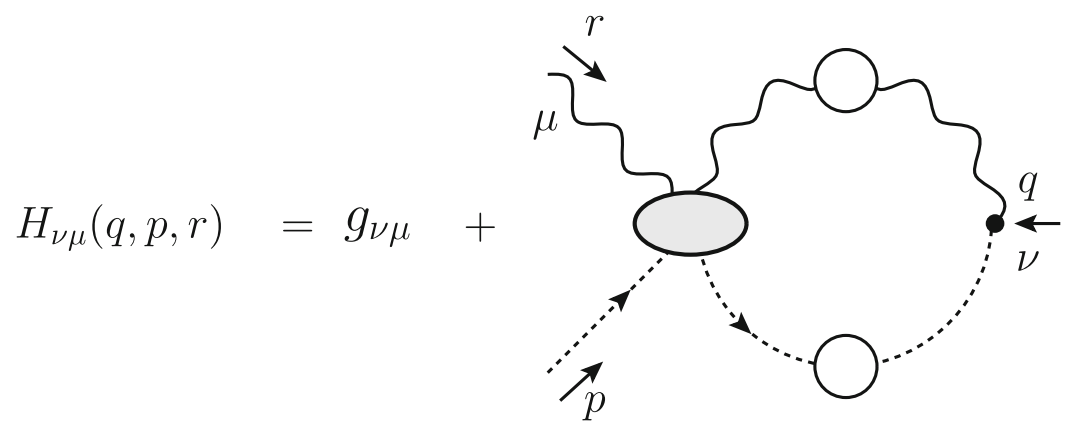

(c)

Fig. 1 Diagrammatic representations of the three-gluon vertex, $\Gamma_{\alpha \mu \nu}^{a b c}(q, r, p)$, the ghost-gluon vertex, $\Gamma_{\mu}^{a b c}(q, p, r)$, and the ghost-gluon kernel, $H_{v \mu}^{a b c}(q, p, r)$ [diagrams $(a),(b)$, and $(c)$, respectively], with the corresponding momentum conventions

114,115,124,125] (Minkowski space)

$$
\begin{aligned}
q^{\alpha} \Gamma_{\alpha \mu \nu}(q, r, p)= & F\left(q^{2}\right)\left[p^{2} J\left(p^{2}\right) \mathrm{P}_{\nu}^{\alpha}(p)\right. \\
& \times H_{\alpha \mu}(p, q, r)-r^{2} J\left(r^{2}\right) \\
& \left.\times \mathrm{P}_{\mu}^{\alpha}(r) H_{\alpha \nu}(r, q, p)\right], \\
q^{\alpha} V_{\alpha \mu \nu}(q, r, p)= & F\left(q^{2}\right)\left[m^{2}\left(r^{2}\right) \mathrm{P}_{\mu}^{\alpha}(r) H_{\alpha \nu}(r, q, p)\right. \\
& \left.-m^{2}\left(p^{2}\right) \mathrm{P}_{\nu}^{\alpha}(p) H_{\alpha \mu}(p, q, r)\right] .
\end{aligned}
$$

Shifting our attention to $\Gamma^{\alpha \mu \nu}(q, r, p)$, it is customary to decompose it in two distinct pieces $[94,95,126]$, according to

$\Gamma^{\alpha \mu \nu}(q, r, p)=\Gamma_{L}^{\alpha \mu \nu}(q, r, p)+\Gamma_{T}^{\alpha \mu \nu}(q, r, p)$,

where the "longitudinal" part, $\Gamma_{L}^{\alpha \mu \nu}(q, r, p)$, saturates Eq. (2.8), while the totally "transverse" part, $\Gamma_{T}^{\alpha \mu \nu}(q, r, p)$, is annihilated when contracted by $q_{\alpha}, r_{\mu}$, or $p_{\nu}$. The tensorial decomposition of these two terms reads $[94-96,126]$

$$
\begin{aligned}
& \Gamma_{L}^{\alpha \mu v}(q, r, p)=\sum_{i=1}^{10} X_{i}(q, r, p) \ell_{i}^{\alpha \mu \nu}, \\
& \Gamma_{T}^{\alpha \mu v}(q, r, p)=\sum_{i=1}^{4} Y_{i}(q, r, p) t_{i}^{\alpha \mu \nu},
\end{aligned}
$$

where the explicit expressions of the basis elements $\ell_{i}^{\alpha \mu \nu}$ and $t_{i}^{\alpha \mu \nu}$ are given in Eqs. (3.4) and (3.6) of [58], respectively. At tree level,

$$
\Gamma_{\alpha \mu \nu}^{(0)}(q, r, p)=(q-r)_{\nu} g_{\alpha \mu}+(r-p)_{\alpha} g_{\mu \nu}+(p-q)_{\mu} g_{\alpha \nu}
$$

As has been explained in detail in [58], the longitudinal part of $\Gamma_{\alpha \mu \nu}(q, r, p)$ may be reconstructed from the STI in Eq. (2.8) through the nonperturbative generalization of the standard BC construction [94]; in particular, the form factors $X_{i}$ may be expressed in terms of $F\left(q^{2}\right), J\left(q^{2}\right), A_{1}(q, r, p)$, $A_{3}(q, r, p)$, and $A_{d}(q, r, p)$ [58]. Note, however, that this construction leaves the transverse form factors, $Y_{i}$, completely undetermined.

\section{The asymmetric configuration}

Of central interest in what follows is the quantity

$$
L(q, r, p)=\frac{W^{\alpha \mu v}(q, r, p) \mathrm{P}_{\alpha^{\prime} \alpha}(q) \mathrm{P}_{\mu^{\prime} \mu}(r) \mathrm{P}_{v^{\prime} v}(p) \Gamma^{\alpha^{\prime} \mu^{\prime} v^{\prime}}(q, r, p)}{W^{\alpha \mu \nu}(q, r, p) W_{\alpha \mu \nu}(q, r, p)},
$$


employed in numerous lattice simulations of the threegluon vertex $[59,63] . W^{\alpha \mu \nu}(q, r, p)$ represents specific tensors, which project out particular components of the $\Gamma^{\alpha^{\prime} \mu^{\prime} v^{\prime}}(q, r, p)$, evaluated in special kinematic limits [59, 63].

In the present work we will focus on the asymmetric limit, corresponding to the kinematic configuration

$p \rightarrow 0, \quad r=-q$.

Then, an appropriate choice for the $W^{\alpha \mu \nu}$ (see Eq. (2.22) of [59]) give rise to the special version of $L(q, r, p)$, denoted by $L^{\text {asym }}\left(q^{2}\right)$, which have been computed on the lattice, in quenched [60-63,92] as well as unquenched [59] simulations.

As has been shown in [58,59], $L^{\text {asym }}\left(q^{2}\right)$ is given by

$L^{\operatorname{asym}}\left(q^{2}\right)=X_{1}(q,-q, 0)-q^{2} X_{3}(q,-q, 0) ;$

since it contains no transverse form factors, $Y_{i}, L^{\text {asym }}\left(q^{2}\right)$ is fully determined from the BC solution for $X_{1}$ and $X_{3}$. The $A_{i}$ entering in this solution appear in two different kinematic configurations, $A_{i}(q,-q, 0)$ and $A_{i}(q, 0,-q)$, corresponding to the soft gluon and soft ghost limits, respectively; we will employ the short-hand notation

$A_{i}\left(q^{2}\right):=A_{i}(q,-q, 0), \quad \widetilde{A}_{i}\left(q^{2}\right):=A_{i}(q, 0,-q)$.

Note that, by virtue of Taylor's theorem [81], in the Landau gauge, $\widetilde{A}_{1}\left(q^{2}\right)$ is a $q^{2}$-independent constant (see "Appendix A" for details); therefore, in what follows we simply set $\widetilde{A}_{1}\left(q^{2}\right) \rightarrow \widetilde{A}_{1}$.

The case of $X_{1}(q,-q, 0)$ can be read off directly from [58]; specifically, after conversion to Euclidean space,

$X_{1}(q,-q, 0)=F\left(q^{2}\right) J\left(q^{2}\right) A_{1}\left(q^{2}\right)$.

The form factor $X_{3}(q, r, p)$ is given by (Minkowski space)

$$
\begin{aligned}
X_{3}(q, r, p)= & \frac{F\left(p^{2}\right)}{q^{2}-r^{2}}\left[J\left(q^{2}\right) G_{1}(q, r, p)\right. \\
& \left.-J\left(r^{2}\right) G_{1}(r, q, p)\right],
\end{aligned}
$$

where

$G_{1}(q, r, p):=A_{1}(q, p, r)+(q \cdot p) A_{d}(q, p, r)$.

Evidently, due to the vanishing of the denominator, the $p=0$ limit requires an appropriate expansion. Using that $q^{2}-r^{2}=-2 q \cdot p+\mathcal{O}\left(p^{2}\right)$, and expanding $J\left(r^{2}\right)$ in the numerator, i.e. $J\left(r^{2}\right)=J\left(q^{2}\right)+2 q \cdot p J^{\prime}\left(q^{2}\right)$, we obtain

$$
\begin{aligned}
X_{3}(q,-q, 0)= & F(0)\left[J^{\prime}\left(q^{2}\right) \widetilde{A}_{1}-J\left(q^{2}\right)\right. \\
& \left.\times\left(\widetilde{A}_{d}\left(q^{2}\right)+G_{3}\left(q^{2}\right)\right)\right], \\
G_{3}\left(q^{2}\right):= & \lim _{p \rightarrow 0} \frac{G_{2}(q, r, p)}{2(q \cdot p)}, \\
G_{2}(q, r, p):= & A_{1}(q, p, r)-A_{1}(r, p, q) .
\end{aligned}
$$

In order to evaluate $G_{3}\left(q^{2}\right)$ further, note that $A_{1}(q, p, r)$ is obtained from $H_{v \mu}(q, p, r)$ through the projection $A_{1}(q, p, r)=$ $\mathcal{T}_{1}^{\mu \nu}(q, r) H_{\nu \mu}(q, p, r)$, where the projector $\mathcal{T}_{1}^{\mu v}(q, r)$ satisfies

$$
\begin{gathered}
\mathcal{T}_{1}^{\mu \nu}(r, q)=\mathcal{T}_{1}^{\mu \nu}(q, r) ; \quad \mathcal{T}_{1}^{\mu}(q, r)=1 ; \\
\mathcal{T}_{1}^{\mu \nu}(q, r) t_{\mu}=0 ; \quad \mathcal{T}_{1}^{\mu \nu}(q,-q)=\frac{\mathrm{P}_{\mu \nu}(q)}{3},
\end{gathered}
$$

with $t=q, r, p$ (see Eq. (3.8) of [69]). Moreover, in the Landau gauge, all quantum corrections to $H_{\nu \mu}(q, p, r)$ are proportional to $p$, such that [125]

$H_{\nu \mu}(q, p, r)=g_{\mu \nu} \widetilde{A}_{1}+p^{\rho} K_{\nu \mu \rho}(q, p, r)$.

Hence, the $G_{2}(q, r, p)$ of Eq. (3.9) may be cast in the form $G_{2}(q, r, p)=p^{\rho} \mathcal{T}_{1}^{\mu v}(q, r)\left[K_{v \mu \rho}(q, p, r)-K_{v \mu \rho}(r, p, q)\right]$.

Evidently, the limit $p=0$ may be taken directly in the expression in square brackets, yielding

$$
\begin{aligned}
G_{2}(q, r, p)= & p^{\rho} \mathcal{T}_{1}^{\mu \nu}(q,-q)\left[K_{\nu \mu \rho}(q, 0,-q)\right. \\
& \left.-K_{\nu \mu \rho}(-q, 0, q)\right]+\mathcal{O}\left(p^{2}\right) .
\end{aligned}
$$

Next, $K_{\nu \mu \rho}(q, 0,-q)$ may be written as

$K_{\nu \mu \rho}(q, 0,-q)=-\frac{\mathcal{W}\left(q^{2}\right)}{q^{2}} g_{\mu \nu} q_{\rho}+\cdots$,

where the ellipses denote terms proportional to $g_{\mu \rho} q_{\nu}, g_{\rho \nu} q_{\mu}$, and $q_{\mu} q_{\nu} q_{\rho}$, which do not contribute to $G_{2}(q, r, p)$, by virtue of Eq. (3.10). Hence, we have

$G_{2}(q, r, p)=-2(q \cdot p) \frac{\mathcal{W}\left(q^{2}\right)}{q^{2}}+\mathcal{O}\left(p^{2}\right)$,

which, upon substitution into Eq. (3.13), leads to $G_{3}\left(q^{2}\right)=$ $-\mathcal{W}\left(q^{2}\right) / q^{2}$. Using the last expression into Eq. (3.8), and passing to Euclidean space $\left(q^{2} \rightarrow-q^{2}\right)$, we finally get

$$
\begin{aligned}
X_{3}(q,-q, 0)= & -F(0) J^{\prime}\left(q^{2}\right) \widetilde{A}_{1}\left(q^{2}\right) \\
& -F(0) J\left(q^{2}\right)\left(\widetilde{A}_{d}\left(q^{2}\right)+\frac{\mathcal{W}\left(q^{2}\right)}{q^{2}}\right) .
\end{aligned}
$$

Substituting Eqs. (3.5) and (3.16) into Eq. (3.3) leads immediately to

$$
\begin{aligned}
L^{\operatorname{asym}}\left(q^{2}\right)= & F\left(q^{2}\right) J\left(q^{2}\right) A_{1}\left(q^{2}\right)+q^{2} F(0)\left[J^{\prime}\left(q^{2}\right) \widetilde{A}_{1}\right. \\
& \left.+\left(\widetilde{A}_{d}\left(q^{2}\right)+\frac{\mathcal{W}\left(q^{2}\right)}{q^{2}}\right) J\left(q^{2}\right)\right] ;
\end{aligned}
$$

the detailed derivation of the function $\mathcal{W}\left(q^{2}\right)$ is given in "Appendix B" (see in particular (B1)).

The above equation may be simplified considerably by resorting to the exact relation

$$
\frac{F\left(r^{2}\right)\left[A_{1}(q, r, p)-p^{2} A_{3}(q, r, p)-(q \cdot p) A_{4}(q, r, p)\right]}{F\left(p^{2}\right)\left[A_{1}(q, p, r)-r^{2} A_{3}(q, p, r)-(q \cdot r) A_{4}(q, p, r)\right]}=1,
$$


which is a direct consequence of a fundamental STI satisfied by $H_{\nu \mu}[69,127]$. In particular, setting in it $p=0$ and $r=$ $-q$, we obtain

$q^{2} \widetilde{A}_{d}\left(q^{2}\right)=\widetilde{A}_{1}-\frac{F\left(q^{2}\right)}{F(0)} A_{1}\left(q^{2}\right)$.

The substitution of the above result into Eq. (3.17) eliminates all dependence on $A_{1}\left(q^{2}\right), \widetilde{A}_{3}\left(q^{2}\right)$, and $\widetilde{A}_{4}\left(q^{2}\right)$, yielding the compact result

$L^{\operatorname{asym}}\left(q^{2}\right)=F(0)\left[J\left(q^{2}\right)\left(\widetilde{A}_{1}+\mathcal{W}\left(q^{2}\right)\right)+q^{2} J^{\prime}\left(q^{2}\right) \widetilde{A}_{1}\right]$.

It is clear from Eq. (3.20) that the logarithmic divergence displayed by $J\left(q^{2}\right)$ in the deep infrared is transferred to $L^{\text {asym }}\left(q^{2}\right)$. In particular, from Eq. (B9) follows that $\mathcal{W}(0)=0$ (for more details, see the end of "Appendix B"); moreover, $\widetilde{A}_{1}=Z_{1}^{\text {asym }}$ (see Eq. (A1) in the "Appendix A"), while the term $\lim _{q^{2} \rightarrow 0} q^{2} J^{\prime}\left(q^{2}\right)$ is subleading, contributing a finite constant. Thus, the leading contribution of Eq. (3.20) is given by

$\lim _{q^{2} \rightarrow 0} L^{\operatorname{asym}}\left(q^{2}\right)=Z_{1}^{\text {asym }} F(0) \lim _{q^{2} \rightarrow 0} J\left(q^{2}\right)$,

relating the rates of divergence of $L^{\text {asym }}\left(q^{2}\right)$ and $J\left(q^{2}\right)$ at the origin.

\section{Derivation of the sum rules}

It is clear that the STI-derived relation given in Eq. (3.20) may be regarded as a first order linear differential equation for $J\left(q^{2}\right)$, whose solution allows one to express $J\left(q^{2}\right)$ in terms of all other functions. It turns out that this particular point of view, when appropriately explored, leads to two novel constraints, whose detailed derivation is the focal point of this section.

\subsection{Asymmetric sum rule}

Let us consider Eq. (3.20), set $x=q^{2}$, and define

$f_{1}(x)=1+\frac{\mathcal{W}(x)}{\widetilde{A}_{1}}, \quad f_{2}(x)=\frac{L^{\operatorname{asym}}(x)}{F(0) \widetilde{A}_{1}}$.

Then, Eq. (3.20) may be cast in the "canonical" form of a linear differential equation

$J^{\prime}(x)+P(x) J(x)=Q(x)$,

with

$P(x)=\frac{f_{1}(x)}{x}, \quad Q(x)=\frac{f_{2}(x)}{x}$.

Therefore, the solution of Eq. (4.2) reads [128]

$J(x)=\frac{1}{\lambda(x)}\left[\lambda\left(\mu^{2}\right) J\left(\mu^{2}\right)+\int_{\mu^{2}}^{x} d t \lambda(t) Q(t)\right]$, where $\lambda(x)$ is the "integrating factor", given by

$\lambda(x)=\exp \left[\int d x P(x)\right]$,

and $\mu^{2}$ is the point where the initial condition is chosen.

At this point, it is natural to opt for an initial condition dictated by the physics, identifying $\mu^{2}$ with the subtraction point where $J(x)$ has been renormalized. Specifically, in the momentum subtraction scheme (MOM) usually employed, we have that $J\left(\mu^{2}\right)=1$, so that Eq. (4.4) becomes

$J(x)=\frac{1}{\lambda(x)}\left[\lambda\left(\mu^{2}\right)+\int_{\mu^{2}}^{x} d t \lambda(t) Q(t)\right]$.

The particularity of this solution originates from the presence of the $x$ in the denominator of $P(x)$, which, in general, introduces in the answer a pole divergence at $x=0$.

To see this property in its most rudimentary manifestation, set into Eq. (4.1) $\mathcal{W}(x)=0$ (tree-level value), so that $f_{1}(x)=1$, while $f_{2}(x)$ is kept arbitrary. Then, the integrating factor becomes simply

$\lambda(x)=x$,

and the solution reads

$J(x)=\frac{1}{x}\left[\mu^{2}+\int_{\mu^{2}}^{x} d t f_{2}(t)\right]$.

Now, let us suppose that we know from independent considerations that $J(x)$ does not diverge as a pole at $x=0$, but rather as a logarithm $[58,59,97]$. Then, the question that arises naturally is how to reconcile this information with the form of Eq. (4.8).

Perhaps the most direct approach for answering this question is to consider the Taylor expansion around $x=0$ of the expression in square brackets on the r.h.s. of Eq. (4.8). Specifically, one has

$\mu^{2}+\int_{\mu^{2}}^{x} d t f_{2}(t)=a_{0}+a_{1} x+\mathcal{O}\left(x^{2}\right)$,

with

$a_{0}=\mu^{2}+\int_{\mu^{2}}^{0} d t f_{2}(t), \quad a_{1}=f_{2}(0)$.

Clearly, in order for the solution not to possess a pole at the origin, we must have $a_{0}=0$. This condition amounts to the integral constraint

$\int_{0}^{\mu^{2}} d t f_{2}(t)=\mu^{2}$

which must be obeyed by the function $f_{2}(t)$ within the interval of integration $\left[0, \mu^{2}\right]$.

If the above condition is satisfied, then the solution of the differential equation at $x=0$ yields $J(0)=f_{2}(0)$, which is none other than the leading term of Eq. (3.21). 
Let us emphasize that the constraint (4.11) does not hinge on the specifics of the behavior of $J(x)$, other than the fact that it does not display an $1 / x$ divergence as $x \rightarrow 0$. For example, regardless of whether $J(x)$ displays near the origin the logarithmic behavior advocated in the literature, or goes simply to a constant, the corresponding $f_{2}(t)$ is bound to satisfy Eq. (4.11).

Having fixed the ideas, let us next consider the complete case, where the function $f_{1}(x)$ retains its full structure. Noting that $\mathcal{W}(0)=0$, we have that $f_{1}(0)=1$; it is then convenient to define

$u(x):=f_{1}(x)-1=\frac{\mathcal{W}(x)}{\widetilde{A}_{1}}$,

with $u(0)=0$. Then, Eq. (4.5) yields

$\lambda(x)=x \sigma(x), \quad \sigma(x):=\exp \left[\int d x u(x) / x\right]$.

As is clear from Eq. (3.11), $H_{\nu \mu}, K_{v \mu \rho}$, and their respective form factors, $\widetilde{A}_{1}$ and $\mathcal{W}\left(q^{2}\right)$, are all renormalized by the the same (finite) constant, $Z_{1}^{\text {asym }}$, which drops out when forming the ratio that defines $u(x)$. Consequently, both $u(x)$ and $\sigma(x)$ are "renormalization group invariant" $(\mu$-independent $)$ quantities.

With the definitions introduced in Eq. (4.13), the solution in Eq. (4.6) becomes

$J(x)=\frac{1}{x \sigma(x)}\left[\mu^{2} \sigma\left(\mu^{2}\right)+\int_{\mu^{2}}^{x} d t \sigma(t) f_{2}(t)\right]$,

and the Taylor expansion around $x=0$ can be carried through as in Eq. (4.9). At this point, the requirement of eliminating from the solution the pole at $x=0$ imposes the constraint

$\int_{0}^{\mu^{2}} d t \sigma(t) f_{2}(t)=\mu^{2} \sigma\left(\mu^{2}\right)$,

which is a central result of this work, to be referred to as the "asymmetric sum rule".

Next, assuming the constraint of Eq. (4.15) to be satisfied, the asymptotic behavior of $J\left(q^{2}\right)$ is obtained from the first nonvanishing term of the Taylor expansion, yielding exactly the leading relation reported in Eq. (3.21).

\subsection{Alternative derivation}

Let us return to Eq. (3.20), but, instead of solving it for $J(x)$, use Eq. (2.2) to set $J(x)=\left[\Delta^{-1}(x)-m^{2}(x)\right] / x$, and convert it into a differential equation for $m^{2}(x)$, treating $\Delta(x)$ as an input known from lattice simulations. Then, straightforward algebra yields

$\left[m^{2}(x)\right]^{\prime}+P_{m}(x) m^{2}(x)=Q_{m}(x)$, with

$P_{m}(x)=\frac{\mathcal{W}(x)}{x \widetilde{A}_{1}}$,

$Q_{m}(x)=-f_{2}(x)+\left[\Delta^{-1}(x)\right]^{\prime}+\frac{\mathcal{W}(x)}{x \widetilde{A}_{1}} \Delta^{-1}(x)$.

It is clear at this point that the integrating factor, $\lambda_{m}(x)$, is given by $\lambda_{m}(x)=\sigma(x)$, where $\sigma(x)$ is defined in Eq. (4.13), and that $\mathcal{W}(x) /\left[x \widetilde{A}_{1}\right]=\sigma^{\prime}(x) / \sigma(x)$, which allows us to write $Q_{m}(x)$ in the form

$Q_{m}(x)=-f_{2}(x)+\frac{\left[\Delta^{-1}(x) \sigma(x)\right]^{\prime}}{\sigma(x)}$.

Then, choosing the boundary condition at the origin, namely $m^{2}(0)=\Delta^{-1}(0)$, and using that $\sigma(0)=1$, the solution for $m^{2}(x)$ is given by

$$
\begin{aligned}
m^{2}(x)= & \frac{1}{\sigma(x)}\left[\Delta^{-1}(0)+\int_{0}^{x} d t \sigma(t) Q_{m}(t)\right] \\
= & \frac{1}{\sigma(x)}\left[\Delta^{-1}(0)+\left[\Delta^{-1}(t) \sigma(t)\right]_{0}^{x}\right. \\
& \left.-\int_{0}^{x} d t \sigma(t) f_{2}(t)\right] \\
= & \Delta^{-1}(x)-\frac{1}{\sigma(x)} \int_{0}^{x} d t \sigma(t) f_{2}(t) .
\end{aligned}
$$

The direct comparison between the last line of Eqs. (4.19) and (2.2) prompts immediately the identification

$J(x)=\frac{1}{x \sigma(x)} \int_{0}^{x} d t \sigma(t) f_{2}(t)$.

If at this point we impose the normalization condition $J\left(\mu^{2}\right)=1$ at the level of Eq. (4.20), we recover directly the constraint (4.15).

At first sight, it would seem that the constraint of Eq. (4.15) has now been obtained without the key assumption that $J(x)$ should not have a pole. Note, however, that this property is implicitly assumed as soon as one uses the relation $m^{2}(0)=$ $\Delta^{-1}(0)$; indeed, if $J(x)$ had a pole, then $x J(x)$ would furnish a constant at the origin, and one could not attribute the value $\Delta^{-1}(0)$ exclusively to $m^{2}(0)$.

We emphasize that Eqs. (4.20) and (4.14) are completely equivalent, as can be seen immediately by using $\int_{\mu^{2}}^{x}=\int_{0}^{\mu^{2}}-\int_{0}^{x}$ in Eq. (4.14) and subsequently employing Eq. (4.15). Furthermore, note that once the solution for $J(x)$ has been cast in the form of Eq. (4.20), it's pole may be explicitly removed by means of the simple change of variables $t=x y$.

\subsection{Symmetric sum rule}

Another special version of the $L(q, r, p)$ defined in Eq. (3.1), to be denoted by $L^{\mathrm{sym}}\left(s^{2}\right)$, corresponds to the totally sym- 
metric limit $[59,63]$,

$q^{2}=p^{2}=r^{2}:=s^{2}, \quad q \cdot p=q \cdot r=p \cdot r=-\frac{s^{2}}{2}$,

with the appropriate projector $W^{\alpha \mu \nu}(q, r, p)$ given by Eq. (2.18) of [59].

$L^{\mathrm{sym}}\left(s^{2}\right)$ receives contributions from both longitudinal and transverse form factors,

$$
\begin{aligned}
L^{\text {sym }}\left(s^{2}\right)= & \underbrace{X_{1}\left(s^{2}\right)-\frac{s^{2}}{2} X_{3}\left(s^{2}\right)}_{L_{L}^{\text {sym }}\left(s^{2}\right)} \\
& +\underbrace{\frac{s^{4}}{4} Y_{1}\left(s^{2}\right)-\frac{s^{2}}{2} Y_{4}\left(s^{2}\right)}_{L_{T}^{\text {sym }}\left(s^{2}\right)} .
\end{aligned}
$$

Thus, using the results for $X_{i}$ obtained in [58], we may express $L_{L}^{\text {sym }}\left(s^{2}\right)$ as

$$
\begin{aligned}
L_{L}^{\text {sym }}\left(s^{2}\right)= & F\left(s^{2}\right)\left[J\left(s^{2}\right)\left(H_{1}\left(s^{2}\right)+\frac{s^{2}}{2} H_{3}\left(s^{2}\right)\right)\right. \\
& \left.+\frac{s^{2}}{2} J^{\prime}\left(s^{2}\right) H_{2}\left(s^{2}\right)\right]
\end{aligned}
$$

where the $H_{i}\left(s^{2}\right)$ are the combinations of the $A_{i}$ and their derivatives given in Eq. (4.2) of [59].

Exactly as Eq. (3.20), this last equation assumes the form of a first order linear differential equation for $J\left(s^{2}\right)$, namely $\left(x=s^{2}\right)$

$J^{\prime}(x)+\bar{P}(x) J(x)=\bar{Q}(x)$,

where

$\bar{P}(x)=\frac{\bar{f}_{1}(x)}{x}, \quad \bar{Q}(x)=\frac{\bar{f}_{2}(x)}{x}$,

and

$\bar{f}_{1}(x)=\frac{2 H_{1}(x)+x H_{3}(x)}{H_{2}(x)}$,

$\bar{f}_{2}(x)=\frac{2\left[L^{\mathrm{sym}}(x)-L_{T}^{\mathrm{sym}}(x)\right]}{H_{2}(x) F(x)}$.

Then, the analysis leading to Eq. (4.15) can be repeated, with some minor adjustments.

From the dynamical equations describing $A_{i}\left(s^{2}\right)$ [69], one may show that at the origin

$H_{1}(0)=H_{2}(0)=Z_{1}^{\mathrm{sym}}, \quad\left[s^{2} H_{3}\left(s^{2}\right)\right]_{s^{2}=0}=0$,

where $Z_{1}^{\text {sym }}$ is the exact analogue of $Z_{1}^{\text {asym }}$. Hence, Eq. (4.26) implies that $\bar{f}_{1}(0)=2$, whereas in the asymmetric case we had $f_{1}(0)=1$; this extra factor of 2 accounts for the double pole encountered below.
Specifically, the solution of Eq. (4.24) reads

$J(x)=\frac{1}{x^{2} \bar{\sigma}(x)}\left[\mu^{4} \bar{\sigma}\left(\mu^{2}\right)+\int_{\mu^{2}}^{x} d t t \bar{\sigma}(t) \bar{f}_{2}(t)\right]$,

where

$\bar{\sigma}(x):=\exp \left[\int d x \bar{u}(x) / x\right], \quad \bar{u}(x):=\bar{f}_{1}(x)-2$,

and $\bar{u}(0)=0$.

The main difference between Eqs. (4.28) and (4.14) is that now the would-be pole in the solution is not simple but double. Therefore, one needs to consider one more term in the corresponding Taylor expansion, namely

$$
\begin{aligned}
& \mu^{4} \bar{\sigma}\left(\mu^{2}\right)+\int_{\mu^{2}}^{x} d t t \bar{\sigma}(t) \bar{f}_{2}(t) \\
& =\bar{a}_{0}+\bar{a}_{1} x+\frac{1}{2} \bar{a}_{2} x^{2}+\mathcal{O}\left(x^{3}\right),
\end{aligned}
$$

with

$\bar{a}_{0}=\mu^{4} \bar{\sigma}\left(\mu^{2}\right)+\int_{\mu^{2}}^{0} d t t \bar{\sigma}(t) \bar{f}_{2}(t)$,

$\bar{a}_{1}=\left[x \bar{\sigma}(x) \bar{f}_{2}(x)\right]_{x=0}$,

$\bar{a}_{2}=\left[\bar{\sigma}(x) \bar{f}_{2}(x)+x \bar{f}_{2}^{\prime}(x) \bar{\sigma}(x)+x \bar{\sigma}^{\prime}(x) \bar{f}_{2}(x)\right]_{x=0}$.

Clearly, in order for the solution not to have a pole (double or simple) at the origin, we must have $\bar{a}_{0}=0$ and $\bar{a}_{1}=0$. The first condition amounts to the integral constraint

$\int_{0}^{\mu^{2}} d t t \bar{\sigma}(t) \bar{f}_{2}(t)=\mu^{4} \bar{\sigma}\left(\mu^{2}\right)$,

which constitutes the second major result of the present study, to be referred to as the "symmetric sum rule".

Given that $\bar{\sigma}(0)=1$, the condition $\bar{a}_{1}=0$ is satisfied as long as $\bar{f}_{2}(x)$ diverges more mildly than $1 / x$; evidently, this is comfortably fulfilled by the physical $\bar{f}_{2}(x)$, which diverges logarithmically at the origin.

If the above two conditions hold, Eq. (4.28) yields $J(0)=$ $\frac{1}{2} \bar{a}_{2}$; its leading contribution is identical (as it should) to that obtained by setting $s^{2}=0$ directly into Eq. (4.23).

\section{Numerical analysis}

In this section, we focus on the sum rule of Eq. (4.15) and analyze in detail how it can be used to restrict the form of $V\left(\ell^{2}\right)$, which is one of the main ingredients entering in the oneloop dressed approximation of the function $\mathcal{W}\left(q^{2}\right)$, given by Eqs. (B9) and (B10). 


\subsection{Setting up the stage}

(i) It is convenient to cast the sum rule (4.15) into the equivalent form

$$
\begin{aligned}
& \int_{0}^{\mu^{2}} d t \mathcal{K}(t)=1, \\
& \mathcal{K}(t):=\frac{\mathcal{R}(t) L^{\operatorname{asym}}(t)}{\mu^{2} Z_{1}^{\text {asym }} F(0)}, \quad \mathcal{R}(t):=\sigma(t) / \sigma\left(\mu^{2}\right) .
\end{aligned}
$$

The quantity $\mathcal{R}(t)$ captures the net effect that different forms of $\sigma(t)$ induce on the kernel $\mathcal{K}(t)$.

(ii) Throughout the analysis, we use $\mu=4.3 \mathrm{GeV}$, and $\alpha_{s}(\mu):=g^{2}(\mu) / 4 \pi=0.27$, as determined by the lattice simulation of [64].

(iii) For $\Delta\left(q^{2}\right)$ and $F\left(q^{2}\right)$, renormalized at the aforementioned $\mu$, we employ the fits given in Eqs. (4.1) and Eq. (6.1) of [69], respectively. Both fits are in excellent agreement with the lattice data of [44]. Note that from Eq. (6.1) of [69], one has $F(0)=2.82$. Moreover, in accordance with the discussion of "Appendix A", we set $\widetilde{A}_{1}=Z_{1}^{\text {asym }} \approx 0.9$.

(iv) For $B_{1}\left(q^{2}\right)$, entering in Eq. (B10), we use the curve shown in Fig. 2, obtained from the numerical solution of the SDE governing the ghost-gluon vertex, evaluated in the "soft-ghost" kinematic limit; see [69] for details. $B_{1}\left(q^{2}\right)$ can be accurately fitted by the functional form

$$
B_{1}\left(q^{2}\right)=1+\alpha_{s}\left[\frac{\omega_{1}\left(q^{2} / \kappa_{1}^{2}\right)^{\gamma_{1}}}{1+\left(q^{2} / \kappa_{1}^{2}\right)^{\gamma_{1}}}+\frac{\omega_{2}\left(q^{2} / \kappa_{2}^{2}\right)}{1+\left(q^{2} / \kappa_{2}^{2}\right)^{\gamma_{2}}}\right],
$$

where the adjustable parameters acquire the following values: $\gamma_{1}=1.128, \gamma_{2}=1.84, \kappa_{1}^{2}=0.101 \mathrm{GeV}^{2}$, $\kappa_{2}^{2}=1.59 \mathrm{GeV}^{2}, \omega_{1}=0.379$, and $\omega_{2}=1.071$.

(v) For $L^{\text {asym }}\left(q^{2}\right)$ we employ a rather good fit to the lattice data of $[63,64]$ whose $\chi^{2} /$ d.o.f $=0.024$. The curve is shown in Fig. 3, and its functional form is given by

$$
\begin{aligned}
L^{\operatorname{asym}}\left(q^{2}\right)= & F\left(q^{2}\right) T\left(q^{2}\right) \\
& \times+v_{1}\left(\frac{1}{1+\left(q^{2} / \nu_{2}\right)^{2}}-\frac{1}{1+\left(\mu^{2} / \nu_{2}\right)^{2}}\right),
\end{aligned}
$$

with

$$
\begin{aligned}
T\left(q^{2}\right)= & +\frac{3 \lambda_{2}}{4 \pi}\left(1+\frac{\tau_{1}}{q^{2}+\tau_{2}}\right) \\
& \times\left[2 \ln \left(\frac{q^{2}+\eta^{2}\left(q^{2}\right)}{\mu^{2}}\right)+\frac{1}{6} \ln \left(\frac{q^{2}}{\mu^{2}}\right)\right],
\end{aligned}
$$

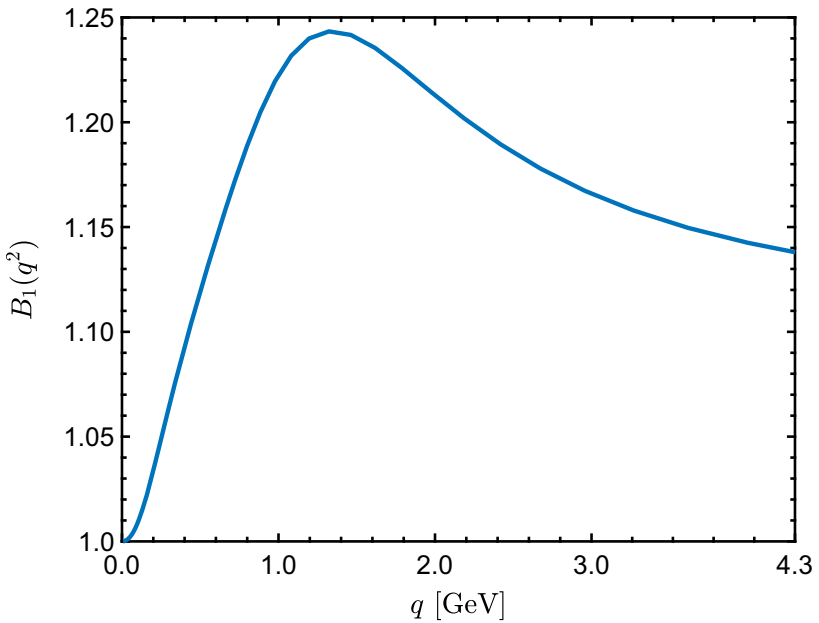

Fig. 2 The ghost-gluon vertex form factor, $B_{1}\left(q^{2}\right)$, given by Eq. (5.2)

and

$\eta^{2}\left(q^{2}\right)=\frac{\xi}{q^{2}+\tau_{0}}$

where the fitting parameters are $\lambda_{2}=0.276, \tau_{0}=0.41$ $\mathrm{GeV}^{2}, \tau_{1}=4.05 \mathrm{GeV}^{2}, \tau_{2}=0.16 \mathrm{GeV}^{2}, \nu_{1}=0.52$, $\nu_{2}=0.012 \mathrm{GeV}^{2}$, and $\xi=10.2 \mathrm{GeV}^{4}$.

Note that the above fit incorporates, by construction, the renormalization condition $L^{\operatorname{asym}}\left(\mu^{2}\right)=1$, corresponding to the "asymmetric" MOM scheme employed. In addition, the zero crossing of $L^{\text {asym }}\left(q^{2}\right)$ is located at $167 \mathrm{MeV}$.

Observe that the above functional form captures the expected infrared asymptotic behavior of Eq. (3.21). In particular, by expanding Eq. (5.3) around $q^{2} \rightarrow 0$, one obtains

$\lim _{q^{2} \rightarrow 0} L^{\operatorname{asym}}\left(q^{2}\right)=a \ln \left(q^{2} / \mu^{2}\right)+b$,

where $a$ and $b$ are constants, comprised by combinations of the fitting parameters entering in the Eq. (5.3); the validity of (5.6) is restricted to the range (080) $\mathrm{MeV}$.

In Fig. 3, the green shaded area is obtained by varying $\xi$ by $\pm 2 \%$, keeping all other fitting parameters fixed; equivalently, at the level of (5.6), this amounts to changing $b$ by $\pm 5 \%$, while $a$ remains unchanged. The spread induced to $L^{\text {asym }}\left(q^{2}\right)$ by this variation is more pronounced in the infrared [ 0.37 at $100 \mathrm{MeV}$ ], being gradually reduced as one moves towards higher momenta (e.g., 0.20 at $300 \mathrm{MeV}$ ).

(vi) To every set of ingredients entering into the sum rule we will assign the corresponding percentage error, denoted 


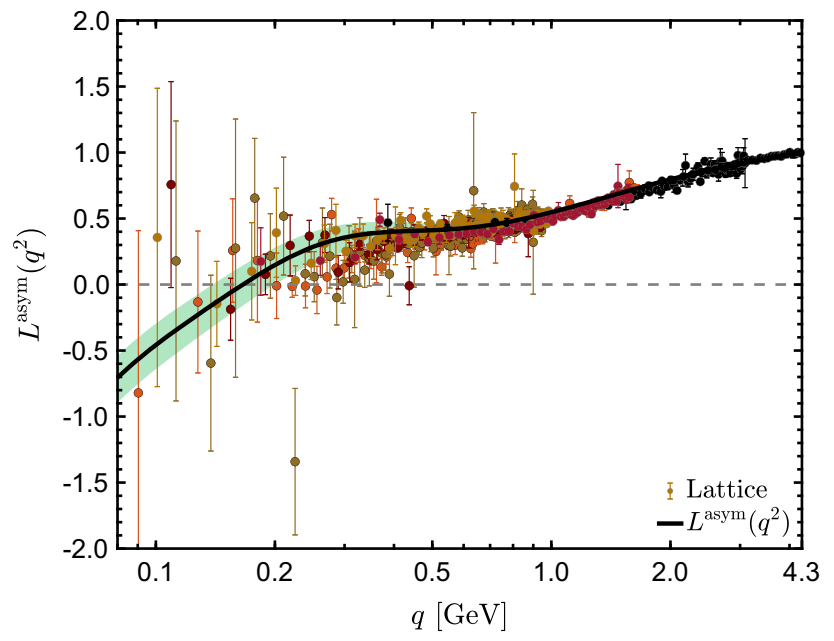

Fig. 3 Lattice data for $L^{\text {asym }}\left(q^{2}\right)$ (circles) from [63,64], and the fit given by Eq. (5.3). The green shaded band shows a spread in the $L^{\text {asym }}\left(q^{2}\right)$ of approximately 0.37 at zero momentum, and reduces to 0.20 at $300 \mathrm{MeV}$

by $\varepsilon$, measuring the deviation of the result from unity, i.e.,

$\varepsilon=\left[\int_{0}^{\mu^{2}} d t \mathcal{K}(t)-1\right] \times 100 \%$.

(vii) The function $\sigma(x)$ is defined in Eq. (4.13) in terms of an indefinite integral; however, if the integration cannot be carried out analytically, one needs to convert it to a definite integral, to be evaluated numerically. This, in turn, introduces an arbitrary integration constant, which amounts to a rescaling of the answer; nonetheless, it is clear from Eqs. (4.28) and (4.32) that such a rescaling is irrelevant, given that $\sigma(x)$ enters homogeneously in the sum rule. In what follows we use the form

$\sigma(x)=\exp \left[\int_{0}^{x} d t u(t) / t\right]$

choosing this particular lower limit of integration fixes the overall scale such that $\sigma(0)=1$.

\subsection{Pinning down $V\left(\ell^{2}\right)$}

We next present a concrete example of how the sum rule of Eq. (5.1), accompanied by a set of physically motivated assumptions, may restrict severely some of the ingredients comprising its kernel. Specifically, we discuss in detail the impact that the variations of the form factor $V\left(\ell^{2}\right)$ have on $\mathcal{W}\left(q^{2}\right)$, and, eventually, through the form of $\sigma\left(q^{2}\right)$, on the sum rule itself. This particular choice is prompted by the numerical exploration of the expressions for $\mathcal{W}_{d_{1}}\left(q^{2}\right)$ and $\mathcal{W}_{d_{2}}\left(q^{2}\right)$, given in Eq. (B10), whose upshot is that $\mathcal{W}_{d_{2}}\left(q^{2}\right)$ furnishes the dominant contribution, and that its value is considerably more sensitive to variations of $V\left(\ell^{2}\right)$ rather than of $B_{1}\left(q^{2}\right)\left[F\left(q^{2}\right)\right.$ and $\Delta\left(q^{2}\right)$ are held fixed]. It is therefore reasonable to establish whether the sum rule is able to place nontrivial bounds on the form and main features exhibited by $V\left(\ell^{2}\right)$.

Let us emphasize at this point that $V\left(\ell^{2}\right)$ emerged at the final step of a series of approximations, described in "Appendix B", whose purpose was to simplify the treatment of the equations defining $\mathcal{W}\left(q^{2}\right)$; it should be therefore interpreted as an "effective" form factor, capturing the collective action of the various $X_{i}$ and $Y_{i}$ comprising the three-gluon vertex, with their multitude of sizes and kinematic dependence. In this sense, there is no a priori guarantee that $V\left(\ell^{2}\right)$ will inherit from them their characteristic suppression in the intermediate region of momenta. Nonetheless, as we will see in what follows, the sum rule clearly favors a "suppressed" $V\left(\ell^{2}\right)$, imposing, at the same time, strict limits on the amount of its suppression.

Next, we introduce a concrete functional form for $V\left(\ell^{2}\right)$, whose variations will generate distinct versions of this quantity. Specifically, we employ the Ansatz

$$
\begin{aligned}
V\left(\ell^{2}\right)= & 1+\frac{\lambda_{s}}{32 \pi}\left(1+\frac{\tau_{1}}{\ell^{2}+\tau_{2}}\right) \\
& \times\left[33 \ln \left(\frac{\ell^{2}+\eta^{2}\left(\ell^{2}\right)}{\mu^{2}}\right)+\ln \left(\frac{\ell^{2}}{\mu^{2}}\right)\right],
\end{aligned}
$$

with $\eta^{2}\left(\ell^{2}\right)$ given by Eq. (5.5). The parameters that will be varied throughout this analysis are $\xi$ and $\tau_{0}$; all others are kept fixed at the values $\lambda_{s}=0.22, \tau_{1}=11.6 \mathrm{GeV}^{2}$, and $\tau_{2}=0.0856 \mathrm{GeV}^{2}$. In Table 1 we quote the sets $\left\{\xi, \tau_{0}\right\}$ that generate the curves shown in Fig. 4. Note that, depending on the choice of the fitting parameters, one may achieve suppression or enhancement in the intermediate region of momenta. Moreover, as commented below, the infrared and ultraviolet limits of this Ansatz are motivated by theoretical considerations.

The starting point of the study consists in establishing the response of the sum rule in two relatively "extreme" situations: $V\left(\ell^{2}\right)=V_{1}\left(\ell^{2}\right)$, showing a substantial enhancement, and $V\left(\ell^{2}\right)=V_{4}\left(\ell^{2}\right)$, displaying a considerable amount of suppression. The substitution of these two versions of $V\left(\ell^{2}\right)$ into the formulas furnishing $\mathcal{W}\left(q^{2}\right)$ (Eqs. (B9) and (B10)), and the subsequent evaluation of Eq. (5.7), reveals that the resulting errors differ in sign: $\varepsilon_{1}>0$, but $\varepsilon_{4}<0$ (see Table 1 ).

Since $\varepsilon$ changes sign when switching from $V\left(\ell^{2}\right)=$ $V_{1}\left(\ell^{2}\right)$ to $V\left(\ell^{2}\right)=V_{4}\left(\ell^{2}\right)$, it is reasonable to expect that there will be an "intermediate" curve, to be denoted by $V_{\star}\left(\ell^{2}\right)$, for which the sum rule will be fulfilled exactly $(\varepsilon=0)$. To determine it, we employ Eq. (5.9) in order to produce a sequence of variations for $V\left(\ell^{2}\right)$, determining each time the corresponding $\varepsilon$; note that the modifications to $V\left(\ell^{2}\right)$ are imple- 
Table 1 The values of the parameters $\xi$ and $\tau_{0}$ used in (5.9) in order to obtain the $V_{i}\left(\ell^{2}\right)$ shown in Fig. 4 . The tree-level case, $V_{3}=1$, is recovered by simply setting $\lambda_{s}=0$. In the last row we quote the percentage error $\varepsilon$, given by Eq. (5.7), when the sum rule (5.1) is computed using the $\sigma_{i}\left(q^{2}\right)$ obtained with the variations of the form factor $V_{i}\left(\ell^{2}\right)$ shown in Fig. 4

\begin{tabular}{|c|c|c|c|c|c|}
\hline$V\left(\ell^{2}\right)$ & $V_{1}\left(\ell^{2}\right)$ & $V_{2}\left(\ell^{2}\right)$ & $V_{3}=1$ & $V_{\star}\left(\ell^{2}\right)$ & $V_{4}\left(\ell^{2}\right)$ \\
\hline$\xi\left(\mathrm{GeV}^{4}\right)$ & 266.4 & 169.5 & - & 99.4 & 24.2 \\
\hline$\tau_{0}\left(\mathrm{GeV}^{2}\right)$ & 10.6 & 7.3 & - & 4.8 & 1.2 \\
\hline$\varepsilon(\%)$ & 24.5 & 13.0 & 9.5 & 0 & -19.5 \\
\hline
\end{tabular}

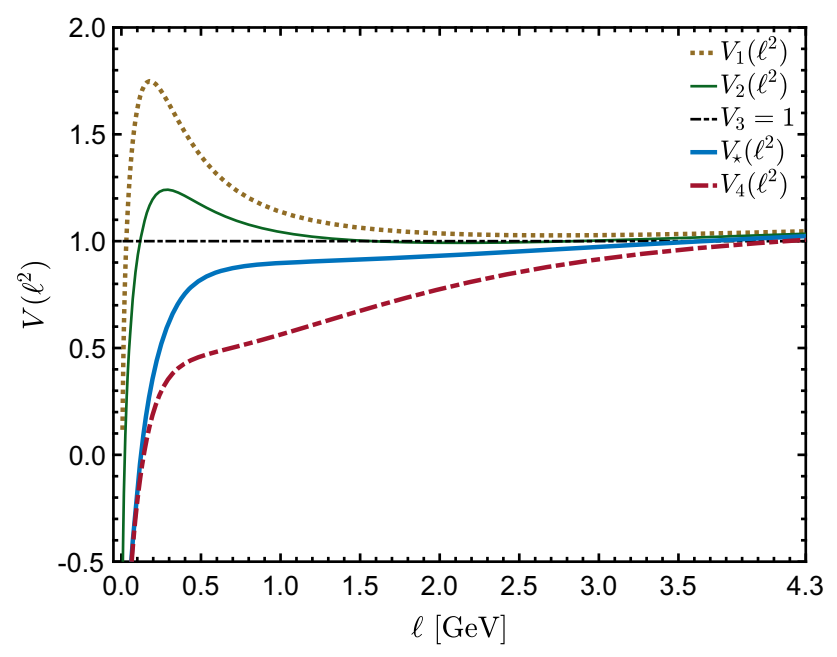

Fig. 4 The five representative cases for $V_{i}\left(\ell^{2}\right)$. All curves are obtained using the the Ansatz given by Eq. (5.9), together with the set of parameters quoted in Table 1

mented in the transition region between nonperturbative and perturbative regimes, i.e., approximately from $300 \mathrm{MeV}$ to $3 \mathrm{GeV}$, as shown in the Fig. 4. All variations considered merge together in the ultraviolet and infrared regimes; specifically, in the ultraviolet they recover the one-loop results for $X_{1}\left(\ell^{2}\right)=X_{4}\left(\ell^{2}\right)=X_{7}\left(\ell^{2}\right)$ [94,95], while in the infrared they diverge at a common logarithmic rate [58].

In Table 1 we show the values for $\varepsilon$ when the sum rule is evaluated using the $\sigma\left(q^{2}\right)$ obtained with the various $V_{i}\left(\ell^{2}\right)$ shown in Fig. 4. Evidently, the sum rule favors clearly a $V\left(\ell^{2}\right)=V_{\star}\left(\ell^{2}\right)$ that is suppressed with respect to the treelevel value captured by $V_{3}$, but certainly less so than the initial $V_{4}\left(\ell^{2}\right)$.

It is rather instructive to analyze in some detail how the above result emerges. To that end, denote by $\left[\mathcal{W}_{1}\left(q^{2}\right)\right.$, $\left.\sigma_{1}\left(q^{2}\right)\right],\left[\mathcal{W}_{\star}\left(q^{2}\right), \sigma_{\star}\left(q^{2}\right)\right]$, and $\left[\mathcal{W}_{4}\left(q^{2}\right), \sigma_{4}\left(q^{2}\right)\right]$, the corresponding quantities obtained when $V\left(\ell^{2}\right)=V_{1}\left(\ell^{2}\right)$, $V\left(\ell^{2}\right)=V_{\star}\left(\ell^{2}\right)$, and $V\left(\ell^{2}\right)=V_{4}\left(\ell^{2}\right)$, respectively. As can be seen in Fig. 5, the suppression induced to $V\left(\ell^{2}\right)$ through the transition $V_{1}\left(\ell^{2}\right) \rightarrow V_{4}\left(\ell^{2}\right)$ leads to an enhancement of $\mathcal{W}\left(q^{2}\right)$ in the region of $800 \mathrm{MeV}-2 \mathrm{GeV}$, where clearly
$\mathcal{W}_{4}\left(q^{2}\right)>\mathcal{W}_{1}\left(q^{2}\right)$. This difference is transmitted to the $\sigma_{1}\left(q^{2}\right)$ and $\sigma_{4}\left(q^{2}\right)$, which, as depicted in Fig. 5, satisfy the relation $\sigma_{4}\left(q^{2}\right)>\sigma_{1}\left(q^{2}\right)$ in the entire range of momenta. Notice that, even though $\mathcal{W}_{1}\left(q^{2}\right)$ and $\mathcal{W}_{4}\left(q^{2}\right)$ merge into each other past $q=4 \mathrm{GeV}, \sigma_{1}\left(q^{2}\right)$ and $\sigma_{4}\left(q^{2}\right)$ remain clearly separated in the same region of momenta; in fact, as can be seen in the inset, they reach their maximum difference precisely at the end point of the momentum interval. This, in turn, indicates that the ultraviolet behavior of $\sigma\left(q^{2}\right)$ is particularly sensitive to the low energy structure of the corresponding $\mathcal{W}\left(q^{2}\right)$. Quite interestingly, as can be seen in Fig. 5, when the corresponding ratios $\mathcal{R}_{1}\left(q^{2}\right)$ and $\mathcal{R}_{4}\left(q^{2}\right)$ are formed, the initial hierarchy $\sigma_{4}\left(q^{2}\right)>\sigma_{1}\left(q^{2}\right)$ is inverted: now $\mathcal{R}_{4}\left(q^{2}\right)<\mathcal{R}_{1}\left(q^{2}\right)$. As a result, the suppressed $V_{4}\left(\ell^{2}\right)$ gives rise to the kernel $\mathcal{K}_{4}(t)$ that is itself suppressed, with respect to $\mathcal{K}_{1}(t)$, in the momentum interval between the zero crossing (at approximately $0.03 \mathrm{GeV}^{2}$ ) and $\mu^{2}$, which practically accounts for the entire value of the sum rule integral. Indeed, the support between the origin and the zero crossing is completely negligible; for example, in the case of $\mathcal{K}_{\star}(t)$, it contributes to the full answer a mere -0.017 . Consequently, the logarithmic divergence encoded into $L^{\text {asym }}\left(q^{2}\right)$ is practically undetected by the sum rule.

Up until this point, the entire analysis has been carried out using a fixed $L^{\text {asym }}\left(q^{2}\right)$, namely the one given by Eq. (5.3), with the values of the parameters quoted below Eq. (5.5). It is clearly important to examine the stability of the result obtained for $V_{\star}\left(\ell^{2}\right)$ when variations in the form of $L^{\text {asym }}\left(q^{2}\right)$ are introduced. To that end, we repeat the previous procedure, using for $L^{\text {asym }}\left(q^{2}\right)$ the two limiting curves that demarcate the green shaded area in Fig. 3; the corresponding $V_{\star}\left(\ell^{2}\right)$ turn out to be particularly close to the original one, forming the narrow blue band shown in Fig. 6 .

In Fig. 6 one may appreciate how the sum rule maps the original uncertainty in $L^{\operatorname{asym}}\left(q^{2}\right)$ (green band) into a corresponding uncertainty in $V_{\star}\left(\ell^{2}\right)$ (blue band). In particular, note that, through the action of the function $\sigma\left(q^{2}\right)$, the maximum separation is shifted from the deep infrared to the physically more interesting range of $300 \mathrm{MeV}-1.3 \mathrm{GeV}$, where the three $V_{\star}\left(\ell^{2}\right)$ reach their maximum mutual discrepancy of about $4 \%$. 

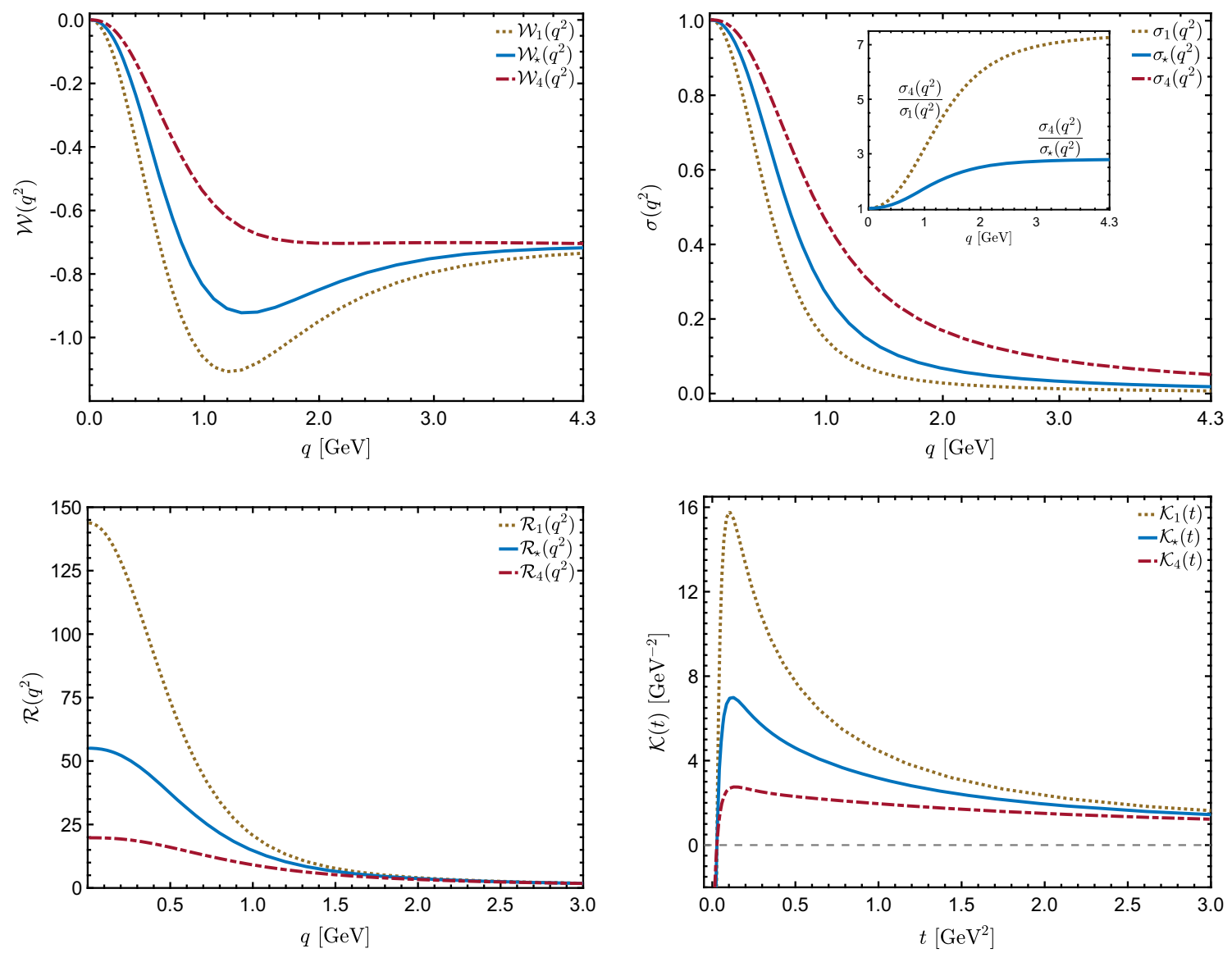

Fig. 5 Top left panel: The functions $\mathcal{W}_{i}\left(q^{2}\right)$ obtained using $V_{1}\left(\ell^{2}\right)$ (brown dotted), $V_{\star}\left(\ell^{2}\right)$ (blue continuous), and $V_{4}\left(\ell^{2}\right)$ (red dasheddotted), shown in Fig. 4. Top right panel: The corresponding $\sigma_{1}\left(q^{2}\right)$,

\section{Discussion and conclusions}

We have presented a couple of new sum rules, which originate from the STIs that connect the two- and three-point sectors of quenched QCD, in the Landau gauge. The key observations that are crucial for their derivation may be summarized as follows. In the context of two special kinematic configurations that involve a single momentum scale, the nonperturbative $\mathrm{BC}$ solutions may be interpreted as exactly solvable ordinary differential equations for the function $J\left(q^{2}\right)$, known as the "kinetic" term of the gluon propagator. The general solutions of these differential equations predict the presence of poles (simple or double) at the origin; however, as dictated by its own SDE, $J\left(q^{2}\right)$ must diverge at the origin only logarithmically. Nonetheless, as an appropriate expansion reveals, these two descriptions may be eventually reconciled, provided that certain integral conditions (sum rules) are exactly satisfied. These sum rules are referred to as "asymmetric" or "symmetric", depending on the kinematic configuration that has served as the starting point for their derivation.

$\sigma_{\star}\left(q^{2}\right)$, and $\sigma_{4}\left(q^{2}\right)$. Bottom left panel: The ratios $\mathcal{R}_{1}\left(q^{2}\right), \mathcal{R}_{\star}\left(q^{2}\right)$, and $\mathcal{R}_{4}\left(q^{2}\right)$, defined in Eq. (5.1). Bottom right panel: The integrands $\mathcal{K}_{1}(t)$, $\mathcal{K}_{\star}(t)$, and $\mathcal{K}_{4}(t)$, given by Eq. $(5.1)$

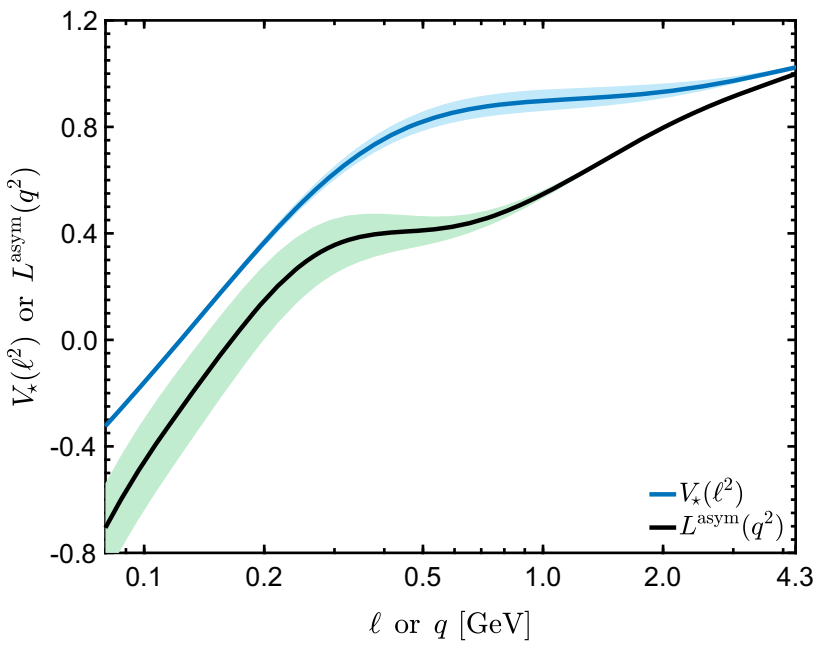

Fig. 6 The propagation of the uncertainty in the $L^{\mathrm{asym}}\left(q^{2}\right)$ to the $V_{\star}\left(\ell^{2}\right)$, through the action of the sum rule

The only element from the two-point sector that enters in these sum rules is the ghost dressing function; its behavior 
is particularly well-established, thanks to large-volume lattice simulation as well as a variety of continuous approaches. All remaining ingredients are related to the three-point sector, whose quantitative exploration, despite the considerable advances mentioned in the Introduction, remains a major technical challenge for the QCD practitioners. The sum rules may serve as a complementary tool in this ongoing quest, furnishing useful constraints for the various components entering in them.

For the purposes of the present work, we have opted for a mixed approach, where certain of the quantities, such as $L^{\text {asym }}\left(q^{2}\right)$, were obtained from the lattice, while the components related to the ghost-gluon kernel from the corresponding SDEs. Alternatively, one may resort to an entirely SDEbased analysis, along the lines of [52,54,55,67,98-100], or to an exclusive functional renormalization group treatment, in the spirit of $[14,38,57]$, such that all relevant quantities are computed within a self-contained framework, and their quality is subsequently assessed by means of the corresponding sum rule. In that vein, one may also envisage a purely latticedriven approach, especially in the context of the asymmetric sum rule; such an effort would entail the simulation of the ghost-gluon kernel, and the extraction of the function $\mathcal{W}\left(q^{2}\right)$.

In the numerical study of Sect. 5 we have used the asymmetric sum rule in order to constrain the effective form factor $V\left(\ell^{2}\right)$, entering in the SDE that determines $\mathcal{W}\left(q^{2}\right)$. In that sense, the sequence $V\left(\ell^{2}\right) \rightarrow \mathcal{W}\left(q^{2}\right) \rightarrow \varepsilon$ was considered, and the "perfect" $V_{\star}\left(\ell^{2}\right)(\varepsilon=0)$ has been determined, which displays a distinct suppression (with respect to unity) in the intermediate range of momenta. It is important to emphasize that no initial bias regarding the suppression of $V\left(\ell^{2}\right)$ was built in; in fact, cases with notable enhancement have been considered $\left(V_{1}, V_{2}\right)$, which, however, were clearly disfavored by the sum rule, as was the case with excessive suppression $\left(V_{4}\right)$. Note finally, that, as mentioned at the end of Sect. 5, the sum rules are completely insensitive to the presence of the zero crossing, and the subsequent logarithmic divergence, displayed by all the $L^{\text {asym }}\left(q^{2}\right)$ considered.

Within the confines of the present approach, the lack of information on $L_{T}^{\text {sym }}\left(s^{2}\right)$ reduces the usefulness of the symmetric sum rule. The situation could be amended if $L_{T}^{\mathrm{sym}}\left(s^{2}\right)$ were to be estimated from the SDE of the three-gluon vertex; in such a case, due to the presence of the extra factor of $t$ in its integrand, the sum rule would be probing the quantities involved predominantly in the intermediate and high momentum regimes.

Finally, it would be particularly interesting to return to the starting point of this investigation, and determine the behavior of the quantity $J\left(q^{2}\right)$ from the solution of the differential equation given in Eq. (4.20). From the conceptual point of view, such a construction may be interpreted as an "inverse" gauge technique, in the sense that, instead of determining a vertex from a propagator, as is usually the case, now the kinetic term of the gluon propagator would be determined from ingredients of the three-point sector of the theory. We expect to present the results of this analysis in the near future.

Acknowledgements The research of J. P. is supported by the Spanish Ministry of Economy and Competitiveness (MINECO) under grant FPA2017-84543-P, and the grant Prometeo/2019/087 of the Generalitat Valenciana. The work of A. C. A. and M. N. F. are supported by the Brazilian National Council for Scientific and Technological Development (CNPq) under the grants 307854/2019-1, 142226/2016-5, and 464898/2014-5 (INCT-FNA). A. C. A. also acknowledges the financial support from the São Paulo Research Foundation (FAPESP) through the project 2017/05685-2.

Data Availability Statement This manuscript has no associated data or the data will not be deposited. [Authors' comment: All data generated or analyzed during this study are included in this published article.]

Open Access This article is licensed under a Creative Commons Attribution 4.0 International License, which permits use, sharing, adaptation, distribution and reproduction in any medium or format, as long as you give appropriate credit to the original author(s) and the source, provide a link to the Creative Commons licence, and indicate if changes were made. The images or other third party material in this article are included in the article's Creative Commons licence, unless indicated otherwise in a credit line to the material. If material is not included in the article's Creative Commons licence and your intended use is not permitted by statutory regulation or exceeds the permitted use, you will need to obtain permission directly from the copyright holder. To view a copy of this licence, visit http://creativecomm ons.org/licenses/by/4.0/.

Funded by $\mathrm{SCOAP}^{3}$.

\section{Appendix A: Changing renormalization schemes}

In this Appendix we address certain technical issues related to the renormalization schemes employed in the computation of the various quantities entering in the sum rule.

(i) The sum rule of Eq. (4.15) has been derived using explicitly the condition $J\left(\mu^{2}\right)=1$, with no further assumptions regarding the renormalization prescription imposed on the remaining quantities. Therefore, it is valid in the context of any self-consistent renormalization scheme that accommodates this particular condition; of course, in practice, one is limited to the few choices used in the literature, such as the various MOM variants, as well as the Taylor scheme.

(ii) Since $J\left(q^{2}\right), F\left(q^{2}\right), H_{\nu \mu}$, and $\Gamma_{\alpha \mu \nu}$ are related by the STI of Eq. (2.8), the number of renormalization conditions that may be chosen freely is reduced down to three. Usually, in addition to $J\left(\mu^{2}\right)=1$, one imposes the condition $F\left(\mu^{2}\right)=1$, because lattice simulations of two point functions opt for this natural condition, and the various functional treatments comply, in order to facilitate the comparison of the results. Moreover, the lattice data for the special projection of 
$\Gamma_{\alpha \mu \nu}$ that we consider have been renormalized such that $L^{\text {asym }}\left(\mu^{2}\right)=1$; we will refer to this particular scheme as "asymmetric" MOM. Thus, at this point, within this particular scheme, the value of the $H_{\nu \mu}$ at the renormalization point $\mu$ is completely fixed by the aforementioned STI.

(iii) The practical upshot of these considerations is that for the numerical evaluation of the sum rules one may not use the $A_{i}$ obtained in [69] together with the lattice results for $L^{\text {asym }}\left(q^{2}\right)$. This is so because in [69] the renormalization was carried out in the Taylor scheme [129], which requires that $H_{\nu \mu}(q, p, r)$ collapses to its tree level value, $g_{v \mu}$ in the soft-ghost kinematics $(p=0)$; however, this value does not coincide with the one obtained from the STI when the asymmetric MOM scheme is employed. To remedy this inconsistency, the $A_{i}$ of [69] must undergo an appropriate rescaling, which will render them compatible with all other inputs.

(iv) As is well-known, the choice of renormalization scheme affects the finite part of the various cutoffdependent renormalization constants, $Z_{i}$, and the transition of the Green's functions from one scheme to the next may be conveniently described as the action of additional finite renormalizations constants. In that sense, the $H_{v \mu}$ calculated in the Landau gauge is special, because its quantum corrections are known to be finite, and do not require an infinite renormalization. Therefore, the corresponding renormalization constant, $Z_{1}$, is finite, and its numerical value may be directly used to describe the transition between the various schemes. Note in particular, that in the Taylor scheme, which will serve as our point of departure, $Z_{1}$ acquires the special value $Z_{1}=1$ [81]. Consequently, the transitions to other schemes will manifest themselves as deviations of $Z_{1}$ from unity. Clearly, the conversion of the $A_{i}$ into the asymmetric MOM scheme requires the use of such a constant, to be denoted by $Z_{1}^{\text {asym }}$.

(v) We next determine approximately the value of $Z_{1}^{\text {asym }}$. Reserving the notation $A_{1}\left(q^{2}\right)$ and $\mathcal{W}\left(q^{2}\right)$ for the quantities defined in the asymmetric scheme, and denoting by $A_{1}^{\mathrm{T}}\left(q^{2}\right)$ and $\mathcal{W}^{\mathrm{T}}\left(q^{2}\right)$ their counterparts in the Taylor scheme, we have

$$
\widetilde{A}_{1}=Z_{1}^{\text {asym }} \widetilde{A}_{1}^{\mathrm{T}}=Z_{1}^{\text {asym }}, \quad \mathcal{W}\left(q^{2}\right)=Z_{1}^{\text {asym }} \mathcal{W}^{\mathrm{T}}\left(q^{2}() \mathrm{A} 1\right)
$$

where we used the special result of the Taylor scheme, $\widetilde{A}_{1}^{\mathrm{T}}=1$.

Then, we set $q^{2}=\mu^{2}$ in Eq. (3.20), and evaluate it in the asymmetric and Taylor schemes; denoting the corresponding results by $L^{\text {asym }}\left(\mu^{2}\right)$ and $\bar{L}^{\text {asym }}\left(\mu^{2}\right)$, respectively, we obtain

$$
\begin{aligned}
& L^{\text {asym }}\left(\mu^{2}\right)=Z_{1}^{\text {asym }} F(0)\left[1+\mathcal{W}^{\mathrm{T}}\left(\mu^{2}\right)+\mu^{2} J^{\prime}\left(\mu^{2}\right)\right], \\
& \bar{L}^{\text {asym }}\left(\mu^{2}\right)=F(0)\left[1+\mathcal{W}^{\mathrm{T}}\left(\mu^{2}\right)+\mu^{2} J^{\prime}\left(\mu^{2}\right)\right] .
\end{aligned}
$$

Since, by definition, $L^{\operatorname{asym}}\left(\mu^{2}\right)=1$, taking the ratio of the two sides of Eq. (A2) yields

$Z_{1}^{\text {asym }}=\frac{1}{\bar{L}^{\text {asym }}\left(\mu^{2}\right)}$.

Next, we assume that the renormalization point $\mu=4.3$ $\mathrm{GeV}$ used in the lattice simulations is sufficiently large for perturbation theory to be a reasonable approximation for $Z_{1}^{\text {asym }}$. Then, the one-loop results for the $X_{i}(q,-q, 0)$ of $[58,95]$, which were computed in the Taylor scheme, can be used to approximate $\bar{L}^{\text {asym }}\left(\mu^{2}\right)$ by

$\bar{L}^{\text {asym }}\left(\mu^{2}\right) \approx 1+\frac{37 C_{\mathrm{A}} \alpha_{s}}{96 \pi}$,

where $C_{\mathrm{A}}$ is the Casimir eigenvalue in the adjoint representation $[N$ for $S U(N)]$. Substituting the above expression in Eq. (A3), yields $\left(\alpha_{s}=0.27\right)$

$Z_{1}^{\text {asym }} \approx 1-\frac{37 C_{\mathrm{A}} \alpha_{s}}{96 \pi} \approx 0.9$.

(v) An exactly analogous discussion holds for the symmetric configuration. The condition $L^{\mathrm{sym}}\left(\mu^{2}\right)=1$, employed for the lattice data in $[59,63]$, defines a scheme that is distinct to the asymmetric MOM, mentioned above; by analogy, it is referred to as the "symmetric" MOM scheme. The conversion of the $A_{i}$ from the Taylor to this latter scheme proceeds by means of a finite renormalization constant, to be denoted by $Z_{1}^{\mathrm{sym}}$, whose numerical value is not required in the present work.

\section{Appendix B: Computing the function $\mathcal{W}\left(q^{2}\right)$}

In this appendix we provide details related with the determination of $\mathcal{W}\left(q^{2}\right)$.

The one-loop dressed approximation of $H_{\nu \mu}$ is obtained from the two diagrams of Fig. 7 [Eq. (3.1) of [69]], which are proportional to the ghost momentum $p$, as can be easily established by contracting the structures $(\ell+p)_{\alpha}$ and $(\ell-q)_{\alpha}$ by the Landau gauge propagators $\Delta_{\alpha \beta}(\ell)$ and $\Delta_{\alpha \beta}(\ell+r)$, respectively; their remainder defines the quantity $K_{v \mu \rho}(q, p, r)$, appearing in the Eq. (3.11), where we set directly $p=0$. Then, from Eq. (3.14) it is straightforward to deduce that $\mathcal{W}\left(q^{2}\right)$ can be extracted from $K_{v \mu \rho}(q, 0,-q)$ through the projection

$\mathcal{W}\left(q^{2}\right)=-\frac{1}{3} q^{\rho} \mathrm{P}^{\mu \nu}(q) K_{\nu \mu \rho}(q, 0,-q)$. 

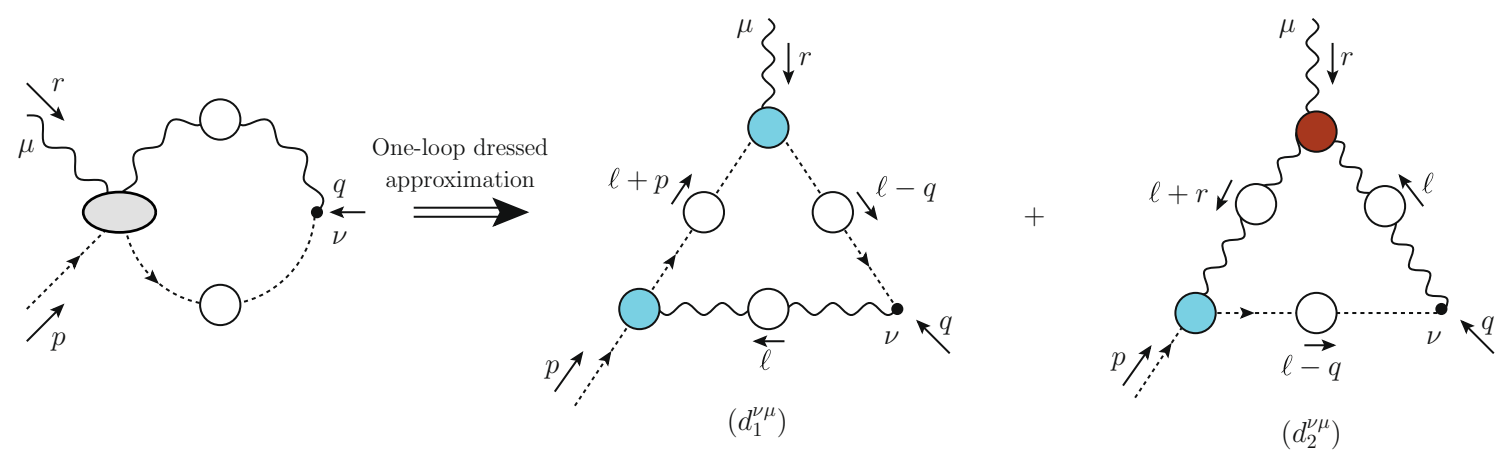

Fig. 7 One-loop dressed approximation of the SDE governing the ghost-gluon scattering kernel

The implementation of Eq. (2.3) at the level of the three ghost-gluon vertices entering in the two diagrams of Fig. 7 reveals that only the form factors $B_{1}$ survive the projection in Eq. (B1). Therefore, the function $B_{2}$ does not contribute to $\mathcal{W}\left(q^{2}\right)$.

In order, to reduce the complexity of the problem, all $B_{1}$ are considered to be functions of one kinematic variable, namely the momentum of the gluon entering into the corresponding ghost-gluon vertex. Specifically, introducing $z:=\ell-q$, this approximation amounts to the replacements

$$
\begin{aligned}
B_{1}(-z, \ell,-q) & \rightarrow B_{1}\left(q^{2}\right), B_{1}(-\ell, 0, \ell) \rightarrow B_{1}\left(\ell^{2}\right), \\
B_{1}(-z, 0, z) & \rightarrow \bar{B}_{1}\left(z^{2}, \ell^{2}\right),
\end{aligned}
$$

where $\bar{B}_{1}\left(z^{2}, \ell^{2}\right):=\frac{1}{2}\left[B_{1}\left(z^{2}\right)+B_{1}\left(\ell^{2}\right)\right]$, originating from the implementation of the symmetrization procedure discussed in Sect. III of [69].

The fully-dressed three-gluon vertex, $\Gamma_{\mu \alpha \beta}(-q,-z, \ell)$, appearing in diagram $\left(d_{2}\right)$, is approximated by its tree-level structure multiplied by a unique form factor, $V$, depending only on $\ell^{2}$, i.e.,

$\Gamma_{\mu \alpha \beta}(-q,-z, \ell)=V\left(\ell^{2}\right) \Gamma_{\mu \alpha \beta}^{(0)}(-q,-z, \ell)$.

With the aforementioned approximations, $K_{v \mu \rho}(q, 0,-q)$ is given by

$$
\begin{aligned}
K_{v \mu \rho}(q, 0,-q)= & \frac{1}{2} C_{\mathrm{A}} g^{2} Z_{1}^{\text {asym }} \\
& \times\left[K_{v \mu \rho}^{d_{1}}(q, 0,-q)+K_{v \mu \rho}^{d_{2}}(q, 0,-q)\right],
\end{aligned}
$$

where $Z_{1}^{\text {asym }}$ is the renormalization constant given in Eq. (A5),

$$
\begin{aligned}
K_{v \mu \rho}^{d_{1}}(q, 0,-q)= & \int_{\ell} \Delta_{\rho v}(\ell) D\left(\ell^{2}\right) \\
& \times D\left(z^{2}\right) B_{1}\left(q^{2}\right) B_{1}\left(\ell^{2}\right)(q-\ell)_{\mu},
\end{aligned}
$$

$$
\begin{aligned}
K_{v \mu \rho}^{d_{2}}(q, 0,-q)= & \int_{\ell} \Delta_{v}^{\beta}(\ell) \Delta_{\rho}^{\alpha}(z) D\left(z^{2}\right) \\
& \times \bar{B}_{1}\left(z^{2}, \ell^{2}\right) V\left(\ell^{2}\right) \Gamma_{\mu \alpha \beta}^{(0)}(-q,-z, \ell),
\end{aligned}
$$

and we resort to the compact notation for the integral over all space

$$
\int_{\ell}:=\frac{1}{(2 \pi)^{4}} \int d^{4} \ell \text {. }
$$

Projecting Eq. (B5) as prescribed in Eq. (B1) leads to

$\mathcal{W}\left(q^{2}\right)=\frac{1}{6} C_{\mathrm{A}} g^{2} Z_{1}^{\text {asym }}\left[\mathcal{W}_{d_{1}}\left(q^{2}\right)+\mathcal{W}_{d_{2}}\left(q^{2}\right)\right]$,

where

$$
\begin{aligned}
\mathcal{W}_{d_{1}}\left(q^{2}\right)= & -i \int_{\ell} \frac{\Delta\left(\ell^{2}\right) F\left(\ell^{2}\right) F\left(z^{2}\right) B_{1}\left(q^{2}\right) B_{1}\left(\ell^{2}\right)}{q^{2} \ell^{4} z^{2}} \\
& \times(\ell \cdot q) h(q, \ell), \\
\mathcal{W}_{d_{2}}\left(q^{2}\right)= & 2 i \int_{\ell} \frac{\Delta\left(\ell^{2}\right) \Delta\left(z^{2}\right) F\left(z^{2}\right) \bar{B}_{1}\left(z^{2}, \ell^{2}\right) V\left(\ell^{2}\right)}{q^{2} \ell^{2} z^{4}} \\
& \times h(q, \ell)\left[z^{2}(\ell \cdot q)+(\ell \cdot q)^{2}+2 q^{2} \ell^{2}\right],
\end{aligned}
$$

with $h(q, \ell):=\ell^{2} q^{2}-(\ell \cdot q)^{2}$.

Finally, we convert Eq. (B7) to Euclidean space, employ spherical coordinates, following the rules and conventions described in Section V of [69]. Note, in particular, that two of the angular integrations may be carried out trivially, furnishing a factor of $4 \pi$; the remaining integration over $\phi$ (the angle between $q$ and $\ell$ ) must be evaluated numerically. The final result reads

$$
\mathcal{W}\left(q^{2}\right)=\frac{C_{\mathrm{A}} \alpha_{s}}{12 \pi^{2}} Z_{1}^{\mathrm{asym}} q\left[\mathcal{W}_{d_{1}}\left(q^{2}\right)+\mathcal{W}_{d_{2}}\left(q^{2}\right)\right],
$$

with $s_{\phi}:=\sin \phi, c_{\phi}:=\cos \phi$, and

$$
\begin{aligned}
\mathcal{W}_{d_{1}}\left(q^{2}\right)= & B_{1}\left(q^{2}\right) \int_{0}^{\infty} d \ell^{2} \ell \Delta\left(\ell^{2}\right) F\left(\ell^{2}\right) B_{1}\left(\ell^{2}\right) \\
& \times \int_{0}^{\pi} d \phi s_{\phi}^{4} c_{\phi} \frac{F\left(z^{2}\right)}{z^{2}},
\end{aligned}
$$




$$
\begin{aligned}
\mathcal{W}_{d_{2}}\left(q^{2}\right)= & -2 \int_{0}^{\infty} d \ell^{2} \ell^{3} \Delta\left(\ell^{2}\right) V\left(\ell^{2}\right) \\
& \times \int_{0}^{\pi} d \phi s_{\phi}^{4}\left(z^{2} c_{\phi}-\ell q s_{\phi}^{2}+3 \ell q\right) \\
& \Delta\left(z^{2}\right) \bar{B}_{1}\left(z^{2}, \ell^{2}\right) \frac{F\left(z^{2}\right)}{z^{4}} .
\end{aligned}
$$

From Eq. (B9) follows immediately that $\mathcal{W}(0)=0$. To estimate at what rate this limit is approached, we resort to a one-loop "massive" calculation, where the infrared finite propagator is replaced by the naive massive $\Delta^{-1}\left(q^{2}\right)=$ $q^{2}-m^{2}$, while all other quantities are kept at their tree level values, $F=B_{1}=\bar{B}_{1}=V=1$. Then, employing standard integration formulas at the level of Eq. (B8), we find that $\lim _{q^{2} \rightarrow 0} \mathcal{W}\left(q^{2}\right) \sim q^{2} \ln \left(q^{2} / m^{2}\right)$.

\section{References}

1. W.J. Marciano, H. Pagels, Phys. Rept. 36, 137 (1978). https://doi. org/10.1016/0370-1573(78)90208-9

2. C.D. Roberts, A.G. Williams, Prog. Part. Nucl. Phys. 33, 477 (1994). https://doi.org/10.1016/0146-6410(94)90049-3

3. R. Alkofer, L. von Smekal, Phys. Rept. 353, 281 (2001). https:// doi.org/10.1016/S0370-1573(01)00010-2

4. P. Maris, C.D. Roberts, Int. J. Mod. Phys. E 12, 297 (2003). https:// doi.org/10.1142/S0218301303001326

5. C.S. Fischer, J. Phys. G 32, R253 (2006). https://doi.org/10.1088/ 0954-3899/32/8/R02

6. C. Roberts, Prog. Part. Nucl. Phys. 61, 50 (2008). https://doi.org/ 10.1016/j.ppnp.2007.12.034

7. D. Binosi, J. Papavassiliou, Phys. Rept. 479, 1 (2009). https://doi. org/10.1016/j.physrep.2009.05.001

8. I.C. Cloet, C.D. Roberts, Prog. Part. Nucl. Phys. 77, 1 (2014)

9. D. Binosi, L. Chang, J. Papavassiliou, C.D. Roberts, Phys. Lett. B 742, 183 (2015). https://doi.org/10.1016/j.physletb.2015.01.031

10. A.C. Aguilar, D. Binosi, J. Papavassiliou, Front. Phys. (Beijing) 11, 111203 (2016a). https://doi.org/10.1007/s11467-015-0517-6

11. D. Binosi, L. Chang, J. Papavassiliou, S.-X. Qin, C.D. Roberts, Phys. Rev. D 93, 096010 (2016). https://doi.org/10.1103/ PhysRevD.93.096010

12. D. Binosi, C. Mezrag, J. Papavassiliou, C.D. Roberts, J. Rodriguez-Quintero, Phys. Rev. D 96, 054026 (2017). https://doi. org/10.1103/PhysRevD.96.054026

13. M.Q. Huber. arXiv: 1808.05227 [hep-ph]

14. J.M. Pawlowski, Ann. Phys. 322, 2831 (2007). https://doi.org/10. 1016/j.aop.2007.01.007

15. J.M. Pawlowski, D.F. Litim, S. Nedelko, L. von Smekal, Phys. Rev. Lett. 93, 152002 (2004). https://doi.org/10.1103/ PhysRevLett.93.152002

16. A. Sternbeck, E.-M. Ilgenfritz, M. Muller-Preussker, A. Schiller, Phys. Rev. D 72, 014507 (2005). https://doi.org/10.1103/ PhysRevD.72.014507

17. E.-M. Ilgenfritz, M. Muller-Preussker, A. Sternbeck, A. Schiller, I. Bogolubsky, Braz. J. Phys. 37, 193 (2007). https://doi.org/10. 1590/S0103-97332007000200006

18. A. Cucchieri, T. Mendes, Phys. Rev. D 78, 094503 (2008a). https:// doi.org/10.1103/PhysRevD.78.094503

19. A. Cucchieri, T. Mendes, Phys. Rev. D 81, 016005 (2010). https:// doi.org/10.1103/PhysRevD.81.016005
20. O. Oliveira, P. Bicudo, J. Phys. G 38, 045003 (2011). https://doi. org/10.1088/0954-3899/38/4/045003

21. A. Maas, Phys. Rept. 524, 203 (2013). https://doi.org/10.1016/j. physrep.2012.11.002

22. P. Boucaud, J.P. Leroy, A.L. Yaouanc, J. Micheli, O. Pene, J. Rodriguez-Quintero, Few Body Syst. 53, 387 (2012). https://doi. org/10.1007/s00601-011-0301-2

23. O. Oliveira, P.J. Silva, Phys. Rev. D 86, 114513 (2012). https:// doi.org/10.1103/PhysRevD.86.114513

24. A.C. Aguilar, J. Papavassiliou, J. High Energy Phys. 12, 012 (2006). https://doi.org/10.1088/1126-6708/2006/12/012

25. A.C. Aguilar, D. Binosi, J. Papavassiliou, Phys. Rev. D 78, 025010 (2008). https://doi.org/10.1103/PhysRevD.78.025010

26. P. Boucaud, J. Leroy, L.Y. A, J. Micheli, O. Pène, J. RodríguezQuintero, J. High Energy Phys. 06, 099 (2008). https://doi.org/ 10.1088/1126-6708/2008/06/099

27. C.S. Fischer, A. Maas, J.M. Pawlowski, Ann. Phys. 324, 2408 (2009). https://doi.org/10.1016/j.aop.2009.07.009

28. M. Tissier, N. Wschebor, Phys. Rev. D 82, 101701 (2010). https:// doi.org/10.1103/PhysRevD.82.101701

29. D.R. Campagnari, H. Reinhardt, Phys. Rev. D 82, 105021 (2010). https://doi.org/10.1103/PhysRevD.82.105021

30. M. Pennington, D. Wilson, Phys. Rev. D 84, 119901 (2011). https://doi.org/10.1103/PhysRevD.84.094028, https:// doi.org/10.1103/PhysRevD.84.119901

31. A.C. Aguilar, D. Ibanez, V. Mathieu, J. Papavassiliou, Phys. Rev. D 85, 014018 (2012). https://doi.org/10.1103/PhysRevD.85. 014018

32. N. Vandersickel, D. Zwanziger, Phys. Rept. 520, 175 (2012). https://doi.org/10.1016/j.physrep.2012.07.003

33. J. Serreau, M. Tissier, Phys. Lett. B 712, 97 (2012). https://doi. org/10.1016/j.physletb.2012.04.041

34. L. Fister, J.M. Pawlowski, Phys. Rev. D 88, 045010 (2013). https:// doi.org/10.1103/PhysRevD.88.045010

35. K.-I. Kondo, S. Kato, A. Shibata, T. Shinohara, Phys. Rept. 579, 1 (2015). https://doi.org/10.1016/j.physrep.2015.03.002

36. M. Tissier, Phys. Lett. B 784, 146 (2018). https://doi.org/10.1016/ j.physletb.2018.07.043

37. L. Corell, A.K. Cyrol, M. Mitter, J.M. Pawlowski, N. Strodthoff, Sci. Post Phys. 5, 066 (2018). https://doi.org/10. 21468/SciPostPhys.5.6.066

38. A.K. Cyrol, M. Mitter, J.M. Pawlowski, N. Strodthoff, Phys. Rev. D 97, 054006 (2018a). https://doi.org/10.1103/PhysRevD. 97.054006

39. F. Gao, S.-X. Qin, C.D. Roberts, J. Rodriguez-Quintero, Phys. Rev. D 97, 034010 (2018). https://doi.org/10.1103/PhysRevD.97. 034010

40. A.K. Cyrol, J.M. Pawlowski, A. Rothkopf, N. Wink, Sci. Post Phys. 5, 065 (2018b). https://doi.org/10.21468/SciPostPhys.5.6. 065

41. W. Kern, M.Q. Huber, R. Alkofer, Phys. Rev. D 100, 094037 (2019). https://doi.org/10.1103/PhysRevD.100.094037

42. A. Cucchieri, T. Mendes, PoS LATTICE2007, 297 (2007). https://doi.org/10.22323/1.042.0297

43. A. Cucchieri, T. Mendes, Phys. Rev. Lett. 100, 241601 (2008b). https://doi.org/10.1103/PhysRevLett.100.241601

44. I. Bogolubsky, E. Ilgenfritz, M. Muller-Preussker, A. Sternbeck, PoS LATTICE2007, 290 (2007). https://doi.org/10.22323/1.042. 0290

45. P.O. Bowman, U.M. Heller, D.B. Leinweber, M.B. Parappilly, A. Sternbeck, L. von Smekal, A.G. Williams, J.-B. Zhang, Phys. Rev. D 76, 094505 (2007). https://doi.org/10.1103/PhysRevD.76. 094505

46. J. Braun, H. Gies, J.M. Pawlowski, Phys. Lett. B 684, 262 (2010), https://doi.org/10.1016/j.physletb.2010.01.009 
47. D. Epple, H. Reinhardt, W. Schleifenbaum, A. Szczepaniak, Phys. Rev. D 77, 085007 (2008). https://doi.org/10.1103/PhysRevD.77. 085007

48. I. Bogolubsky, E. Ilgenfritz, M. Muller-Preussker, A. Sternbeck, Phys. Lett. B 676, 69 (2009). https://doi.org/10.1016/j.physletb. 2009.04.076

49. O. Oliveira, P. Silva, PoS 2, 226 (2009). https://doi.org/10.22323/ 1.091 .0226

50. A. Ayala, A. Bashir, D. Binosi, M. Cristoforetti, J. RodriguezQuintero, Phys. Rev. D 86, 074512 (2012). https://doi.org/10. 1103/PhysRevD.86.074512

51. P. Bicudo, D. Binosi, N. Cardoso, O. Oliveira, P.J. Silva, Phys. Rev. D 92, 114514 (2015). https://doi.org/10.1103/PhysRevD.92. 114514

52. M.Q. Huber, L. von Smekal, J. High Energy Phys. 04, 149 (2013). https://doi.org/10.1007/JHEP04(2013)149

53. M. Pelaez, M. Tissier, N. Wschebor, Phys. Rev. D 88, 125003 (2013). https://doi.org/10.1103/PhysRevD.88.125003

54. A. Blum, M.Q. Huber, M. Mitter, L. von Smekal, Phys. Rev. D 89, 061703 (2014). https://doi.org/10.1103/PhysRevD.88.125003

55. G. Eichmann, R. Williams, R. Alkofer, M. Vujinovic, Phys. Rev. D 89, 105014 (2014). https://doi.org/10.1103/PhysRevD.89. 105014

56. M. Vujinovic, R. Alkofer, G. Eichmann, R. Williams, Acta Phys. Polon. Supp. 7, 607 (2014). https://doi.org/10.5506/ APhysPolBSupp.7.607

57. A.K. Cyrol, L. Fister, M. Mitter, J.M. Pawlowski, N. Strodthoff, Phys. Rev. D 94, 054005 (2016). https://doi.org/10.1103/ PhysRevD.94.054005

58. A.C. Aguilar, M.N. Ferreira, C.T. Figueiredo, J. Papavassiliou, Phys. Rev. D 99, 094010 (2019a). https://doi.org/10.1103/ PhysRevD.99.094010

59. A.C. Aguilar, F. De Soto, M.N. Ferreira, J. Papavassiliou, J. Rodríguez-Quintero, S. Zafeiropoulos, Eur. Phys. J. C 80, 154 (2020). https://doi.org/10.1103/PhysRevD.99.094010

60. A. Cucchieri, A. Maas, T. Mendes, Phys. Rev. D 74, 014503 (2006). https://doi.org/10.1103/PhysRevD.74.014503

61. A. Cucchieri, A. Maas, T. Mendes, Phys. Rev. D 77, 094510 (2008). https://doi.org/10.1103/PhysRevD.77.094510

62. A.G. Duarte, O. Oliveira, P.J. Silva, Phys. Rev. D 94, 074502 (2016). https://doi.org/10.1103/PhysRevD.94.074502

63. A. Athenodorou, D. Binosi, P. Boucaud, F. De Soto, J. Papavassiliou, J. Rodriguez-Quintero, S. Zafeiropoulos, Phys. Lett. D 761, 444 (2016). https://doi.org/10.1016/j.physletb.2016.08.065

64. P. Boucaud, F. De Soto, J. Rodríguez-Quintero, S. Zafeiropoulos, Phys. Rev. D 95, 114503 (2017). https://doi.org/10.1103/ PhysRevD.95.114503

65. P. Boucaud, D. Dudal, J. Leroy, O. Pene, J. Rodriguez-Quintero, J. High Energy Phys. 12, 018 (2011). https://doi.org/10.1007/ JHEP12(2011)018

66. D. Dudal, O. Oliveira, J. Rodriguez-Quintero, Phys. Rev. D 86, 105005 (2012). https://doi.org/10.1103/PhysRevD.86.105005, https://doi.org/10.1103/PhysRevD.86.109902

67. A.C. Aguilar, D. Ibañez, J. Papavassiliou, Phys. Rev. D 87, 114020 (2013). https://doi.org/10.1103/PhysRevD.87.114020

68. B.W. Mintz, L.F. Palhares, S.P. Sorella, A.D. Pereira, Phys. Rev. D 97, 034020 (2018). https://doi.org/10.1103/PhysRevD.97. 034020

69. A.C. Aguilar, M.N. Ferreira, C.T. Figueiredo, J. Papavassiliou, Phys. Rev. D 99, 034026 (2019b). https://doi.org/10.1103/ PhysRevD.99.034026

70. A. Cucchieri, T. Mendes, A. Mihara, J. High Energy Phys. 12, 012 (2004). https://doi.org/10.1088/1126-6708/2004/12/012

71. G. Eichmann, I.C. Cloet, R. Alkofer, A. Krassnigg, C.D. Roberts, Phys. Rev. C 79, 012202 (2009). https://doi.org/10.1103/ PhysRevC.79.012202
72. I. Cloet, G. Eichmann, B. El-Bennich, T. Klahn, C. Roberts, Few Body Syst. 46, 1 (2009). https://doi.org/10.1007/ s00601-009-0015-x

73. C.A. Meyer, E.S. Swanson, Prog. Part. Nucl. Phys. 82, 21 (2015). https://doi.org/10.1016/j.ppnp.2015.03.001

74. G. Eichmann, H. Sanchis-Alepuz, R. Williams, R. Alkofer, C.S. Fischer, Prog. Part. Nucl. Phys. 91, 1 (2016). https://doi.org/10. 1016/j.ppnp.2016.07.001

75. H. Sanchis-Alepuz, R. Alkofer, C.S. Fischer, Eur. Phys. J. A 54 41 (2018). https://doi.org/10.1140/epja/i2018-12465-x

76. R. Alkofer, A. Maas, W.A. Mian, M. Mitter, J. París-López, J.M. Pawlowski, N. Wink, Phys. Rev. D 99, 054029 (2019). https://doi. org/10.1103/PhysRevD.99.054029

77. E.V. Souza, M.N. Ferreira, A.C. Aguilar, J. Papavassiliou, C.D. Roberts, S.-S. Xu, Eur. Phys. J. A 56, 25 (2020). https://doi.org/ 10.1140/epja/s10050-020-00041-y

78. S.-S. Xu, Z.-F. Cui, L. Chang, J. Papavassiliou, C.D. Roberts, H.S. Zong, Eur. Phys. J. A 55, 113 (2019). https://doi.org/10.1140/ epja/i2019-12805-4

79. A.C. Aguilar et al., Eur. Phys. J. A 55, 190 (2019c). https://doi. org/10.1140/epja/i2019-12885-0

80. M.Q. Huber, C.S. Fischer, H. Sanchis-Alepuz. arXiv:2004.00415 [hep-ph]

81. J. Taylor, Nucl. Phys. B 33, 436 (1971). https://doi.org/10.1016/ 0550-3213(71)90297-5

82. A. Slavnov, Theor. Math. Phys. 10, 99 (1972). https://doi.org/10. 1007/BF01090719

83. E. Bagan, T.G. Steele, Phys. Lett. B 219, 497 (1989). https://doi. org/10.1016/0370-2693(89)91101-5

84. P. Boucaud, J. Leroy, A. Le Yaouanc, A. Lokhov, J. Micheli, O. Pene, J. Rodriguez-Quintero, C. Roiesnel, Eur. Phys. J. A 31, 750 (2007). https://doi.org/10.1140/epja/i2006-10295-1

85. A.C. Aguilar, J. Papavassiliou, Phys. Rev. D 83, 014013 (2011). https://doi.org/10.1103/PhysRevD.83.014013

86. O. Oliveira, T. Frederico, W. de Paula, J.P.B.C. de Melo, Eur. Phys. J. C 78, 553 (2018). https://doi.org/10.1140/epjc/ s10052-018-6037-0

87. J. Gracey, H. Kißler, D. Kreimer, Phys. Rev. D 100, 085001 (2019). https://doi.org/10.1103/PhysRevD.100.085001

88. C. Parrinello, Phys. Rev. D 50, R4247 (1994). https://doi.org/10. 1103/PhysRevD.50.R4247

89. B. Alles, D. Henty, H. Panagopoulos, C. Parrinello, C. Pittori, D.G. Richards, Nucl. Phys. B 502, 325 (1997). https://doi.org/10. 1016/S0550-3213(97)00483-5

90. C. Parrinello, D. Richards, B. Alles, H. Panagopoulos, C. Pittori (UKQCD), Nucl. Phys. B Proc. Suppl. 63, 245 (1998). https:// doi.org/10.1016/S0920-5632(97)00734-2

91. P. Boucaud, J.P. Leroy, J. Micheli, O. Pene, C. Roiesnel, J. High Energy Phys. 10, 017 (1998). https://doi.org/10.1088/1126-6708/ 1998/10/017

92. P. Boucaud, F. De Soto, K. Raya, J. Rodríguez-Quintero, S. Zafeiropoulos, Phys. Rev. D 98, 114515 (2018). https://doi.org/ 10.1103/PhysRevD.98.114515

93. S. Zafeiropoulos, P. Boucaud, F. De Soto, J. Rodríguez-Quintero, J. Segovia, Phys. Rev. Lett. 122, 162002 (2019). https://doi.org/ 10.1103/PhysRevLett.122.162002

94. J.S. Ball, T.-W. Chiu, Phys. Rev. D 22, 2550 (1980). https:// doi.org/10.1103/PhysRevD.22.2550 [Erratum: Phys. Rev. D 23, 3085 (1981)]

95. A.I. Davydychev, P. Osland, O. Tarasov, Phys. Rev. D 54, 4087 (1996). https://doi.org/10.1103/PhysRevD.59.109901 [Erratum: Phys. Rev. D 59, 109901 (1999)]

96. A.C. Aguilar, M.N. Ferreira, C.T. Figueiredo, J. Papavassiliou, Phys. Rev. D 100, 094039 (2019d). https://doi.org/10.1103/ PhysRevD.100.094039 
97. A.C. Aguilar, D. Binosi, D. Ibañez, J. Papavassiliou, Phys. Rev. D 89, 085008 (2014). https://doi.org/10.1103/PhysRevD.89. 085008

98. W. Schleifenbaum, A. Maas, J. Wambach, R. Alkofer, Phys. Rev. D 72, 014017 (2005). https://doi.org/10.1103/PhysRevD.72. 014017

99. M.Q. Huber, A. Maas, L. von Smekal, J. High Energy Phys. 11, 035 (2012). https://doi.org/10.1007/JHEP11(2012)035

100. R. Williams, C.S. Fischer, W. Heupel, Phys. Rev. D 93, 034026 (2016). https://doi.org/10.1103/PhysRevD.93.034026

101. A.C. Aguilar, D. Binosi, J. Papavassiliou, Phys. Rev. D 84, 085026 (2011). https://doi.org/10.1103/PhysRevD.84.085026

102. D. Binosi, D. Ibañez, J. Papavassiliou, Phys. Rev. D 86, 085033 (2012). https://doi.org/10.1103/PhysRevD.86.085033

103. J. Smit, Phys. Rev. D 10, 2473 (1974). https://doi.org/10.1103/ PhysRevD.10.2473

104. J.M. Cornwall, Phys. Rev. D 26, 1453 (1982). https://doi.org/10. 1103/PhysRevD.26.1453

105. C.W. Bernard, Phys. Lett. B 108, 431 (1982). https://doi.org/10. 1016/0370-2693(82)91228-X

106. C.W. Bernard, Nucl. Phys. B 219, 341 (1983). https://doi.org/10. 1016/0550-3213(83)90645-4

107. J.F. Donoghue, Phys. Rev. D 29, 2559 (1984). https://doi.org/10. 1103/PhysRevD.29.2559

108. J. Mandula, M. Ogilvie, Phys. Lett. B 185, 127 (1987). https:// doi.org/10.1016/0370-2693(87)91541-3

109. J.M. Cornwall, J. Papavassiliou, Phys. Rev. D 40, 3474 (1989). https://doi.org/10.1103/PhysRevD.40.3474

110. K.G. Wilson, T.S. Walhout, A. Harindranath, W.-M. Zhang, R.J. Perry, S.D. Glazek, Phys. Rev. D 49, 6720 (1994). https://doi.org/ 10.1103/PhysRevD.49.6720

111. O. Philipsen, Nucl. Phys. B 628, 167 (2002). https://doi.org/10. 1016/S0550-3213(02)00089-5

112. A.C. Aguilar, A.A. Natale, P.S. Rodrigues da Silva, Phys. Rev. Lett. 90, 152001 (2003). https://doi.org/10.1103/PhysRevLett.90. 152001

113. A.C. Aguilar, A.A. Natale, J. High Energy Phys. 08, 057 (2004). https://doi.org/10.1088/1126-6708/2004/08/057

114. A.C. Aguilar, D. Binosi, C.T. Figueiredo, J. Papavassiliou, Phys. Rev. D 94, 045002 (2016b). https://doi.org/10.1103/PhysRevD. 94.045002
115. D. Binosi, J. Papavassiliou, Phys. Rev. D 97, 054029 (2018). https://doi.org/10.1103/PhysRevD.97.054029

116. D. Dudal, J.A. Gracey, S.P. Sorella, N. Vandersickel, H. Verschelde, Phys. Rev. D 78, 065047 (2008). https://doi.org/10.1103/ PhysRevD.78.065047

117. L. Abbott, Nucl. Phys. B 185, 189 (1981). https://doi.org/10.1016/ 0550-3213(81)90371-0

118. R. Jackiw, K. Johnson, Phys. Rev. D 8, 2386 (1973). https://doi. org/10.1103/PhysRevD.8.2386

119. J.M. Cornwall, R. Norton, Phys. Rev. D 8, 3338 (1973). https:// doi.org/10.1103/PhysRevD.8.3338

120. E. Eichten, F. Feinberg, Phys. Rev. D 10, 3254 (1974). https://doi. org/10.1103/PhysRevD.10.3254

121. E. Poggio, E. Tomboulis, S.-H. Tye, Phys. Rev. D 11, 2839 (1975). https://doi.org/10.1103/PhysRevD.11.2839

122. J.S. Schwinger, Phys. Rev. 125, 397 (1962a). https://doi.org/10. 1103/PhysRev.125.397

123. J.S. Schwinger, Phys. Rev. 128, 2425 (1962b). https://doi.org/10. 1103/PhysRev.128.2425

124. A.C. Aguilar, D. Binosi, C.T. Figueiredo, J. Papavassiliou, Eur. Phys. J. C 78, 181 (2018). https://doi.org/10.1140/epjc/ s10052-018-5679-2

125. D. Ibañez, J. Papavassiliou, Phys. Rev. D 87, 034008 (2013). https://doi.org/10.1103/PhysRevD.87.034008

126. J. Gracey, Phys. Rev. D 90, 025014 (2014). https://doi.org/10. 1103/PhysRevD.90.025014

127. D. Binosi, J. Papavassiliou, J. High Energy Phys. 03, 121 (2011). https://doi.org/10.1007/JHEP03(2011)121

128. G.B. Arfken, H.J. Weber, F.E. Harris, Mathematical methods for physicists, 7th edn. (Academic Press, San Diego, 2012), pp. 11220

129. P. Boucaud, F. De Soto, J. Leroy, A. Le Yaouanc, J. Micheli et al., Phys. Rev. D 79, 014508 (2009). https://doi.org/10.1103/ PhysRevD.79.014508 